\title{
Accounting Measurement Basis, Market Mispricing, AND Firm Investment EfFICIENCY*
}

\author{
Pierre Jinghong LiAng \\ Tepper School of Business \\ Tech and Frew Streets \\ Carnegie Mellon University \\ Pittsburgh, PA 15213-3890 \\ liangj@andrew. cmu .edu
}

\author{
XIAOYAN WEN \\ Tepper School of Business \\ Tech and Frew Streets \\ Carnegie Mellon University \\ Pittsburgh, PA 15213-3890 \\ xwen@andrew. cmu .edu
}

Current Draft: February 2006

\begin{abstract}
In this paper, we investigate how the accounting measurement basis affects the capital market pricing of a firm's shares, which, in turn, affects the efficiency of the firm's investment decisions. We distinguish two broad bases for accounting measurements: input-based and output-based accounting. We argue that the structural difference in the two measurement bases leads to a systematic difference in the efficiency of the investment decisions. In particular, we show that an output-based measure has a natural advantage in aligning investment incentives because of its comprehensiveness. The (first-) best investment is achieved when the output-based measure is noiseless and manipulation-free. In addition, under an output-based measure, more accounting noise/manipulation always leads to more inefficient investment choices. Therefore, if an outputbased measures is highly noisy and easy to manipulate in practice, the induced investment efficiency can be quite low. On the other hand, an input-based measure, while not as comprehensive, may induce more efficient investment decisions than an output-based measure if some noise is unavoidable in either measure. The reason is two-fold. First, input-based measures may be associated with less noise and limited manipulation in practice. Second and more importantly, we show that under an input-based measure, a slight increase in accounting noise/manipulation may lead to more efficient investment choices. In fact, the (first-) best result is achieved when the noise/manipulability is small but positive. In other words, for an input-based measure, being less comprehensive makes small but positive accounting noise/manipulability desirable. Two extensions of the basic model are also explored.
\end{abstract}

\footnotetext{
${ }^{*}$ The authors gratefully acknowledge the suggestions from an anonymous referee, Joel Demski, Jon Glover, Burton Hollifield, Volker Laux, and members of an informal reading group at the Tepper School of Business, Carnegie Mellon University. All errors are ours. The current version of the paper is available at http://www.tepper.cmu.edu/andrew/liangj.
} 


\section{Introduction}

In this paper, we investigate how the accounting measurement basis affects the capital market pricing of a firm's shares, which, in turn, affects the efficiency of the firm's investment decisions. We distinguish two broad bases for accounting measurements. The first, an output-based accounting measurement, is designed to measure a firm's activities by recording the estimated financial benefits of production. In contrast, the second basis, an input-based accounting measurement, is designed to measure a firm's activities by recording the estimated factor costs of production. We argue that the two bases give rise to different informational properties of accounting numbers. In turn, the different properties induce different capital market pricing, which leads to a systematic difference in the efficiency of the investment decisions. In particular, we show that an output-based measure has a natural advantage in aligning firm investment incentives. That is, because an output-based measure provides comprehensive information about both the scale and profitability of the investment, it reduces the negative impact of mispricing on investment efficiency through a dampening effect. However, output-based measures suffer a potential disadvantage in that they may inherit more measurement noise and may be subjected to more managerial manipulation. We show that an output-based measure performs (first-) best when it is noiseless and manipulation-free. However, in cases where they are highly noisy and easy to manipulate, we show that the investment efficiency can be quite low as a result.

On the other hand, if some noise is unavoidable in any measure, an input-based measure may induce more efficient investment decisions than output-based measures, despite having a disadvantage of only providing information about the scale of the firm investment. The reason is two-fold. First, input-based measures are typically associated with less noise and limited manipulation. Second and more importantly, we show that with an input-based measure, some low levels of noise and manipulability are tolerated or even preferred in some cases. In fact, the (first-) best result is achieved when the measurement noise is small but positive. In other words, for an input-based measure, being less comprehensive makes small but positive measurement noise desirable.

The debate on measurement bases has a long and varied standing in accounting history. Paton and Littleton (1940) describe accounting numbers as price-aggregates which measure the economic activities of a firm. The debate on the basis of price-aggregates centers on which "price" to use in such measurements. Extensive subsequent writings describe the rationales for using entry prices, exit prices, past and/or future market prices, and simulated prices (see Edwards and Bell 1961, Sterling 1970, Ijiri 1975). We frame the debate within a partial equilibrium investment setting with rational expectations and evaluate the different measurement bases on the scale of induced investment efficiency. In other words, we adopt an information-economic paradigm and evaluate the alternative measurements according to their information content. (See Christensen and Demski 2002 for more on such a paradigm.)

The insights in this paper may be helpful in current discussions of fair value measurements. 
There has been considerable movement in public accounting policy toward measurements based on fair value. The move is partially motivated by the desire to increase the relevance of accounting, arguably with some sacrifice in reliability. The emerging concern is the introduction of considerably more estimates which are to be used in preparing fair value accounting measures. The Financial Accounting Standards Board (FASB) has recognized the reliability issue and has prescribed different levels of estimates (Level 1, 2, 3 etc.) accordingly. In this paper, we point to the effects of noise and manipulation on investment efficiency. In particular, we show that using output-based measures makes noise or manipulability a social "bad" to the economy because either of the two induces inefficient investments. In other words, one must always be careful about its negative "real" effect on firm investments if the measure brings in an increased level of noise and manipulability. This is because under output-based measures, more accounting noise/manipulation always leads to more inefficient investment choices. On the other hand, with input-based accounting, the (first-) best result is achieved when the measurement noise is small but positive. As a result, our analysis points to a certain attractiveness of an input-based measurement basis when some measurement noise is unavoidable for either measure. Further, a slight increase in accounting noise/manipulation may lead to more efficient investment choices. Generally, our results imply that the rational economic choice between an output-based measure and an input-based measure is not obvious in most cases where varying levels of noise and manipulability exist in both measures.

Specifically, we consider a simple two-period model in which a firm's investment decision is jointly affected by the total return of the investment and by the short-term capital market pricing of its ownership shares. Conditional on private information about the profitability of the investment, the firm makes an investment decision which generates cash flows in both the first and second periods. The firm's objective is to maximize a weighted average of the life-time cash flows and the short-term share price. The share price reflects the rational expectation of firm value based on a public report of the first-period aggregate cash flow (i.e., the sum of first-period investment return and the cash flow from the first-period on-going activities). The market's inability to identify the sources of the first-period cash flow may induce a systematic short-term mispricing of firm value, from the firm's perspective. In turn, this mispricing induces a sub-optimal investment decision, ex ante. We show that the deviation from the first-best investment level may be positive (i.e., overinvestment) or negative (i.e., under-investment) depending, in part, on the timing of the investment return.

Within this framework, accounting is introduced as an information system which provides (priceaggregate) measures about the investment. In pricing the firm's shares, the capital market uses the information in the accounting measure as a supplement to the information contained in the aggregate cash flow. We consider two accounting measurement bases. First, an output-based accounting measure is modeled as an unbiased estimate of the investment return (which is a function of the actual investment made and the true investment profitability). Second and alternatively, 
an input-based accounting measure is modeled as an unbiased estimate of the actual investment made. Either accounting signal provides additional information, which may help the capital market improve the pricing of the firm's shares. The improved pricing induces better firm investment decisions. As the quality of accounting measures (which is inversely related to the measurement noise) improves, one would expect less mispricing, which would lead to better investment decisions. We show that this conjecture is, indeed, true for both the input- and output-based measures when the noise in these measures is high. That is, when accounting measures are highly imprecise, any improvement in precision will lead to more efficient investment decisions.

When the measurement noise is low, however, the two measurement bases are fundamentally different. For the output-based measure, investment efficiency continues to rise with any increase in measurement quality. At the limit, when the output-based accounting measure perfectly reveals the investment return, first-best investment choices are made. However, for the input-based measure with low measurement noise, the investment efficiency is an inverted U-shape function of the measurement noise. As measurement noise decreases, the investment efficiency first increases up to a threshold point and decreases afterwards. The first-best investment choices are made when the noise is small but not zero (at the threshold point).

The reason for the difference, and for the surprising result, is the different mispricing structures that are generated by the two measurement bases. For the output-based measure, both the actual investment made and the actual investment profitability affect the accounting measure. As the firm deviates from the first-best investment level, the effect on its share price is dampened, at the margin, by the measure's built-in profitability estimate. In other words, the firm's ability to use real investment to change the market perception of its investment profitability is mitigated by the independent profitability estimate built into the output-based measure. In turn, the dampening effect lessens the ex ante incentives to distort the investment decision. The investment efficiency increases monotonically as the measurement noise decreases. For the input-based measure, the accounting measure relies only on the actual investment made and does not directly rely on a separate estimate of the investment profitability. The dampening effect does not apply to the (mis)pricing of the accounting report; it only applies to the (mis)pricing of a portion of the cash flow measure. As the measurement noise decreases, the dampening effect is diminished as more valuation weight is shifted from the cash flow to the accounting report. This is the reason that a noiseless input-based measure invites severe deviation from the first-best investment.

Finally, we offer two extensions of the model. First, we add accounting manipulation to the mix. Following Dye and Sridhar (2004a), we model accounting manipulation by giving the firm an option to privately modify accounting measurements before they are reported to the capital market. The total cost of manipulation contains a random element. As a result, accounting manipulation introduces additional noise into the reported accounting measures. Based on the existing economic forces, it is shown that accounting manipulation always makes the firm worse off 
under an output-based accounting regime because adding more noise to an output-based accounting measure always leads to a less efficient investment decision. However, accounting manipulation may make the firm better off under an input-based accounting regime. This is because there are regions in the inverted U-shape relation where a slight increase in measurement noise will improve the investment efficiency. This is most likely when the existing measurement noise is small, and the noise introduced by accounting manipulation to the input-based measure is also small.

In the second extension, we modify the model to allow an option for the date- 2 shareholders to utilize the technology in the firm to generate future cash flows. In this modified model, the market price is reflective of two streams of cashflows: those generated by the initial investment and those generated by future investments. As before, the mispricing affects the initial investment choice and the relative performance of the input- vs. output-based accounting measures. However, it is no longer true that the output-based measure performs best when noiseless. Comparing accounting measurement bases is further complicated and requires knowing more details of the context including, in particular, the importance of future cash flow relative to current cash flow.

The antecedent of studies on investment myopia in finance and economics is Holmstrom (1982) and Stein (1989). Stein (1989) finds that the managers, facing stock market pressure, forsake good investments so as to boost current earnings, even though the market is efficient and is not fooled in equilibrium. In spite of being unable to fool the market, managers are trapped into behaving myopically in the classic signal-jamming model. Dye and Sridhar (2004a) examine how investment decisions are affected by the reliable and relevant components of an aggregate accounting report. Their study focuses on the reliability-relevance trade-offs in accounting aggregation, the conditions under which aggregation improves efficiency, and on the optimal weights in constructing an optimal accounting report. While Dye and Sridhar (2004a) and our study share the feature of accounting manipulation, our focus is on the comparison of two alternative accounting measurements, each of which is disaggregated from a common cash flow measure. In addition, Demski, Lin, and Sappington (2005) analyze a setting in which entrepreneurs invest before they learn whether they will be forced to sell their assets. They study the optimal design of asset impairment regulations when the assets resale market suffers from the "lemon" problem.

Using a contracting setting, Prendergast (2002) examines input-based and output-based measures and argues that input-based monitoring, coupled with a directed action, performs best in stable settings, while output-based monitoring is best in uncertain environments. The reason is that output-based contracts, coupled with a delegation of decisions, align social and private incentives better in uncertain situations. In contrast, our study focuses on the market incentives produced by input- and output-based measures and finds that the output-based measure performs best when the measurement is precise. Among other accounting studies on investment efficiency, Kanodia, Singh and Spero (2005) analyze the economic consequences of the interaction between noisy accounting measures and information asymmetry regarding the investment profitability. They find that some 
degree of accounting imprecision can be value-enhancing, which is consistent with our results on the input-based measure. In contrast, we focus on the comparison of input- vs. output-based measures and show that the role of accounting imprecision depends on the accounting basis: under an output-based measure, noiseless accounting is optimal.

The rest of the paper proceeds as follows. Section 2 describes the model details. Section 3 presents the benchmark results of the basic setup where only an aggregate cash flow is reported. In section 4, we introduce accounting measures and analyze the equilibria induced by accounting. Section 5 introduces an extension which models accounting manipulation. Section 6 offers another extension where the firm technology is reusable in the future. Section 7 concludes the paper.

\section{The Model}

Consider an economy with a risk-neutral firm in a competitive risk-neutral capital market. There are two periods (and three dates), representing short- and long-term concerns. The firm's normal on-going activities generate a pair of cash flows, denoted $x_{t}$, realized on date- $t(t=1,2)$. We assume:

$$
\left[\begin{array}{l}
x_{1} \\
x_{2}
\end{array}\right] \sim N\left(\left[\begin{array}{l}
\mu \\
\mu
\end{array}\right],\left[\begin{array}{cc}
\sigma^{2} & \alpha \sigma^{2} \\
\alpha \sigma^{2} & \sigma^{2}
\end{array}\right]\right)
$$

On date-0, the firm is faced with an investment opportunity (called a project) and observes a private signal, denoted $\theta \in \mathbb{R}$, about the project's profitability. The prior distribution of $\theta$ is normal with mean $\theta_{0}$ and variance $\sigma_{\theta}^{2}$ (i.e., $\theta \backsim N\left[\theta_{0}, \sigma_{\theta}^{2}\right]$ ). The firm chooses an investment level, denoted $I \in \mathbb{R}^{+}$, to invest into the project. The project generates cash flows on date- 1 and date2 , denoted by $f_{1}(\theta, I)$ and $f_{2}(\theta, I)$ respectively. For tractability, we assume the project returns $f_{1}(\theta, I)=k \sqrt{\theta I}$ and $f_{2}(\theta, I)=(2-k) \sqrt{\theta I}$, where $k \in(0,2)$. Thus, the total investment return $(2 \sqrt{\theta I})$ depends only on the investment level $I$ and the profitability variable $\theta$. The timing of the investment return depends on $k$ : higher $k$ indicates that more investment return is realized in the short-term. ${ }^{1}$

The realized periodic cash flows to the firm are denoted by $z_{t}(t=1,2):^{2}$

$$
\begin{aligned}
& z_{1}=x_{1}+f_{1}(\theta, I)=x_{1}+k \sqrt{\theta I} \\
& z_{2}=x_{2}+f_{2}(\theta, I)=x_{2}+(2-k) \sqrt{\theta I}
\end{aligned}
$$

Following the literature (Dye 2002, Dye and Sridhar 2004a, 2004b), we assume that the investment is made privately (i.e., not directly observable) and that the firm is unable to directly

\footnotetext{
${ }^{1}$ All qualitative results remain if we assume that $f_{t}($.$) are the expected values of the short- and long-term investment$ returns and that the noise in the returns is independent of the existing random variables.

${ }^{2}$ Here we assume that $z_{1}$ is gross of the investment cost $(I)$, which is appropriate given that we assume the investment is made privately. If $z_{1}$ is net of the investment cost, the quantitative results would change while the same qualitative forces would remain. See Dye 2002 and Dye and Sridhar 2004a for similar assumptions.
} 
communicate the private information $(\theta)$ to outsiders, including the capital market. We also assume that $\theta$ is independent of $x_{1}$ or $x_{2}$ and the on-going cash flows $\left(x_{1}\right.$ or $\left.x_{2}\right)$ are not affected by the investment choice $(I)$.

At the end of date 1, the firm's shares are priced in a competitive risk-neutral capital market such that the market price, denoted $P$, is equal to the expected value of the cumulative cash flow on date-2. Denote the publicly available information set on date- 1 by $\Omega$ (assuming no discounting or dividend payments):

$$
P=E\left[z_{1}+z_{2} \mid \Omega\right]
$$

The information set $\Omega$ includes public information available for pricing. In the basic setup, the firm publicly reports its aggregate cash flow only, so $\Omega$ contains $z_{1}$ alone. Later, we consider additional signals created by an accounting information system; so $\Omega$ may include additional items such as a deferral or an accrual.

The firm is motivated by both the long-term interest (i.e., cumulative cash flows on date-2) and the short-term interest (i.e., date-1 stock price). We assume for life cycle or liquidity reasons, a portion of the firm, denoted $\beta \in(0,1)$, must be sold on date-1. The remaining $(1-\beta)$ portion will be held by the date- 0 shareholders. ${ }^{3}$ As a result, the firm's objective is to maximize a weighted average of date- 1 market price and date- 2 cumulative cash flow (net of the investment costs $I$ ). For a type- $\theta$ firm with investment function $I(\theta)$, the objective function is

$$
-I(\theta)+\beta P+(1-\beta)\left(z_{1}+z_{2}\right)
$$

The sequence of the events is summarized below.

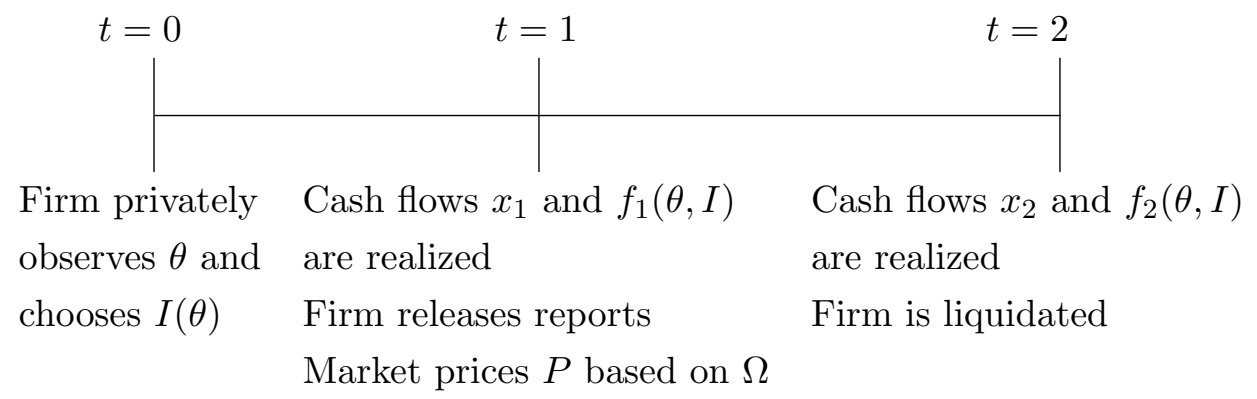

Figure 1: Time Line of Events

As a reference point for what follows, we provide a description of the first-best investment policy in Lemma 1 (proof omitted).

\footnotetext{
${ }^{3}$ Another reason may be that the firm faces a probability $\beta$ of takeover on date- 1 , in which case the firm wishes to maximize its date-1 share price (see Stein 1989 for additional discussions). In the finance literature, incentive to underinvest may be driven by a debt-overhang problem (see Myers 1977).
} 
Lemma 1 (first-best) The socially optimal investment policy consists of a function $I^{F B}(\theta)$, where for each $\theta, I^{F B}(\theta)$ maximizes $f_{1}(\theta, I)+f_{2}(\theta, I)-I=2 \sqrt{\theta I}-I$. So we have $I^{F B}=\theta$ when $\theta>0$; and $I^{F B}=0$ when $\theta<0$.

We now define the equilibrium where the investment policy is made in the self-interest of the firm.

Definition 1 An equilibrium relative to $\Omega$ consists of an investment function $I^{*}(\cdot)$, and a market pricing function $P(\cdot)$, such that:

(i) Given $P(\cdot)$, optimal investment function $I^{*}(\cdot)$ maximizes $V(\theta \mid I(\cdot))=E_{x_{1} x_{2}}[-I+\beta P(\cdot)+$ $\left.(1-\beta)\left(z_{1}+z_{2}\right)\right]$

(ii) Given $I^{*}(\cdot)$, the pricing function $P(\cdot)$ satisfies $P=E\left[z_{1}+z_{2} \mid \Omega, I^{*}(\cdot)\right]$.

We employ an approximation assumption to calculate the pricing function in closed-form.

Approximation Assumption: Let random variables $x$ and $y$ be jointly normally distributed, and $z$ is normally distributed and independent of $x$ and $y$. Denote by $f(x, z \mid y+a z)$ the jointly conditional density function of $x$ and $z$ for some known constant $a$ and denote $G(z \mid y+a z)$ the conditional cumulative density function of $z$. We assume for all realizations of $y+a z$,

$$
\int_{z>0} \int_{x}(x+z) f(x, z \mid y+a z) d x d z+G(0 \mid y+a z) \int_{x} x f(x \mid y) d x \simeq \int_{z} \int_{x}(x+z) f(x, z \mid y+a z) d x d z
$$

In words, the approximation assumes that the error due to censoring the lower tail of a normally distributed random variable is small when calculating the conditional mean of the sum of the censored variable and another normal random variable. In our context, the investment strategy function $I(\theta)$ censors the underlying profitability parameter $\theta$ by the fact $I=0$ if $\theta<0$. The market observes some linear function of $I(\theta)$, not the uncensored $\theta$. The assumption allows us to calculate the market inference in closed-form by assuming the censored $\theta$ is close enough to the uncensored $\theta$. Because the censoring point is always held at zero, it is clear that this approximation becomes increasingly accurate as $E[z]$ is large, i.e., the probability mass to the left of zero is increasingly small. ${ }^{4} 5$

\footnotetext{
${ }^{4}$ Note this approximation assumption pertains to calculating the conditional expectation (i.e., expected $x+z$ conditional on the realization of $y+a z)$. We acknowledge that this is a global requirement. That is, the approximation applies for each and every realization of $y+a z$. For extremely negative realizations of the censored variable $z$, the approximation error may be large. However, the approximation error for a particular realization of $y+a z$, denoted $A E(y+a z)$, becomes smaller and approaches zero as the mean of $z$ increases. In the appendix, we formally prove this claim.

${ }^{5}$ Dye and Sridhar (2004b) use an approximation assumption in their analysis. While their approximation applies to calculating the unconditional mean of an altered normally distributed random variable, ours applies to a series of conditional mean of an altered normally distributed random variable. Stocken and Verrecchia (2004) also uses a similar approximation.
} 


\section{Basic Setup}

Except in Section 6, we assume throughout that on date- 1 the realized aggregate cash flow $\left(z_{1}\right)$ is always publicly observable. However, outsiders are not able to differentiate the cash flow components. In other words, cash flows generated from on-going activities and from the investment are aggregated. This aggregation feature plays an important role in this setting.

We now analyze the equilibrium behavior of the firm in the basic setup.

Theorem 1 Using the approximation assumption (6), there exists a unique linear equilibrium relative to $\Omega=\left\{z_{1}\right\}$. It is given by

(i) an equilibrium linear pricing function:

$$
\begin{aligned}
P\left(z_{1}\right) & =a+b \times z_{1}, \text { where } \\
b & =\frac{(1+\alpha) \sigma^{2}+2 k \delta \sigma_{\theta}^{2}}{\sigma^{2}+k^{2} \delta \sigma_{\theta}^{2}} \\
a & =(2-b) \mu+(2-k b) \sqrt{\delta} \theta_{0}
\end{aligned}
$$

(ii) an equilibrium investment function:

$$
\begin{gathered}
I(\theta)=\left\{\begin{array}{cc}
\delta \theta, & \text { if } \theta \geq 0 \\
0 & \text { if } \theta<0
\end{array}\right. \\
\text { where } \delta=\left(1+\beta\left(\frac{b k}{2}-1\right)\right)^{2}
\end{gathered}
$$

Proof of Theorem 1. All proofs are placed in the appendix.

Compared with the first-best investment, a key observation in Theorem 1 is that the equilibrium investment choice $(I)$ is generally a function of the short-term market pressure (parameter $\beta$ ) and the timing of the investment cash flows (parameter $k$ ), not just a function of the investment profitability (variable $\theta$ ). This is because in pricing the firm's shares, the market is unable to distinguish the individual components of the short-term cash flow $\left(z_{1}\right)$. To illustrate, suppose that the market is able to distinguish the cash flow components; then the appropriate response to every dollar of the on-going cash flows $\left(x_{1}\right)$ would be $(1+\alpha)$ and the appropriate response to the short-term investment return $\left(f_{1}(\theta, I)=k \sqrt{\theta I}\right)$ would be $\frac{2}{k}$. However, because only the aggregate $\left(z_{1}=x_{1}+k \sqrt{\theta I}\right)$ is reported, the market response is the weighted average of $(1+\alpha)$ and $\frac{2}{k}$. Therefore, the market may not price the investment efficiently. Because the firm cares about the short-term share price $(\beta>0)$, the efficiency of the pricing affects the investment incentives.

To understand the nature of the mispricing, we return to the firm objective function (5), which 
may be rewritten as

$$
\begin{aligned}
& -I+\beta P+(1-\beta)\left(z_{1}+z_{2}\right) \\
& =-I+z_{1}+z_{2}+\beta\left(P-\left(z_{1}+z_{2}\right)\right) \\
& =\left(-I+x_{1}+x_{2}+2 \sqrt{\theta I}\right)+\beta\left(P-\left(x_{1}+x_{2}+2 \sqrt{\theta I}\right)\right)
\end{aligned}
$$

The first term of (12) is the first-best objective function. The second term of (12), when $\beta>0$, is an increasing function of the mispricing (equal to price minus the long-term firm value). When making the investment decision $(I)$, the firm does not know $x_{t}$ but knows $\theta$. So, the expected mispricing, given $\theta$, is

$$
E_{x_{1} x_{2}}\left[P-\left(x_{1}+x_{2}+2 \sqrt{\theta I}\right) \mid \theta\right]
$$

Substituting the linear pricing function $P=a+b\left(x_{1}+k \sqrt{\theta I}\right)$ into the expected mispricing, we have

$$
\begin{aligned}
& E_{x_{1} x_{2}}\left[P-\left(x_{1}+x_{2}+2 \sqrt{\theta I}\right) \mid \theta\right] \\
& =E_{x_{1} x_{2}}\left[a+b\left(x_{1}+k \sqrt{\theta I}\right)-\left(x_{1}+x_{2}+2 \sqrt{\theta I}\right) \mid \theta\right] \\
& =(b k-2) \sqrt{\theta I}+E_{x_{1} x_{2}}\left[a+b x_{1}-\left(x_{1}+x_{2}\right)\right]
\end{aligned}
$$

Given that the second term of (13) is not a function of $I$, the mispricing will only affect the equilibrium investment through the first term of $(13):{ }^{6}(b k-2) \sqrt{\theta I}$. So we have

$$
\begin{aligned}
V(\theta \mid I(\cdot)) & =E_{x_{1} x_{2}}\left[\left(-I+x_{1}+x_{2}+2 \sqrt{\theta I}\right)+\beta\left(P-\left(x_{1}+x_{2}+2 \sqrt{\theta I}\right)\right) \mid \theta\right] \\
& =-I+E_{x_{1} x_{2}}\left[x_{1}+x_{2}\right]+2 \sqrt{\theta I}+\beta(b k-2) \sqrt{\theta I}+\beta E_{x_{1} x_{2}}\left[a+b x_{1}-\left(x_{1}+x_{2}\right)\right] \\
& =-I+2 \sqrt{\theta I}+\beta(b k-2) \sqrt{\theta I}+(2+\beta(b-2)) \mu+\beta a
\end{aligned}
$$

When the firm either does not care about the short-term share price $(\beta=0)$ or the market correctly prices firm investments $\left(b=\frac{2}{k}\right)$, the firm makes the first-best investment choices. If neither condition is met, the (mis)pricing is affected by the firm's investment choice, so the firm has an incentive to deviate from the first-best level. In fact, the firm faces conflicting incentives when making its investment decision:

- Incentive to underinvest. Given $k \neq 2$, some investment returns are realized in the longrun. Given $0<\beta<1$, the firm receives only a fraction (i.e., $(1-\beta)$ share) of the long-run marginal benefit but must bear the full marginal cost. This leads to underinvestment because

\footnotetext{
${ }^{6}$ Notice that the second term of (13), while not affected by investment $(I)$, is still a mispricing caused by reporting the aggregate cash flow. This mispricing would induce (operating) inefficiencies if the firm is able to control the timing of the on-going cash flows $\left(x_{1}\right.$ and $\left.x_{2}\right)$. See Stein 1989.
} 
the marginal return is positive but decreasing in $I$. (See similar economic forces in Dye 2002, Dye and Sridhar 2004a, 2004b.)

- Incentive to overinvest. Given $k \neq 0$, some investment returns are realized in the short-run. Given $0<\beta<1$, the firm has an incentive to inflate the first period cash flow $\left(z_{1}=x_{1}+k \sqrt{\theta I}\right)$ because the pricing function places a positive weight on $z_{1}$. In our model, the only way to inflate $z_{1}$ is to increase investment level $(I)$. This leads to overinvestment as the short-run marginal benefit from the investment may be inflated. (See a similar tension in Stein 1989.)

We summarize the investment results with the following theorem.

Theorem 2 In the basic setup $\Omega=\left\{z_{1}\right\}$, there exists a value for $k$, namely, $k^{*}=\frac{2}{1+\alpha}$, such that the linear equilibrium produces the first-best investment level $\delta=1$. If $k>k^{*}$, then $\delta>1$ or the firm overinvests; if $k<k^{*}$, then $\delta<1$, or the firm underinvests.

Intuitively, when $k=k^{*}$, the appropriate response to the short-term investment return $\left(\frac{2}{k}\right)$ happens to be the same as the appropriate response to the on-going cash flow $(1+\alpha)$. That is, the proportion of the short-term investment return perfectly matches the time-series correlation of cash flows from on-going activities. Thus, even with an aggregated cash flows report, the market price motivates the efficient investment decision.

If $k \neq k^{*}$, the market reaction to the aggregate cash flow is an average of $\frac{2}{k}$ and $(1+\alpha)$. When $k>k^{*}$, the average is higher than $\frac{2}{k}$, which places too high of a weight on the short-term investment return, leading to over-investment. When $k<k^{*}$, the average is lower, leading to under-investment. In other words, the aggregation of information leads to market mispricing of the firm investment, which leads to sub-optimal investment decisions.

Using this basic model, we can show that (a) both the market response coefficient $b$ and the investment coefficient $\delta$ increases as $\alpha$ increases; (b) if $k<k^{*}$, both $b$ and $\delta$ increases as the on-going cash flows are less noisy (i.e., $\sigma^{2}$ decreases); and (c) as the short term pressure $\beta$ increases, market response $b$ decreases and firm investment coefficient decreases if $k<k^{*}$. (See Proposition 1 in the appendix for details.)

\section{Accounting}

Now we expand the information set which is available to the market. We are particularly interested in how information contained in the non-cash component of the financial statements affects the market pricing and the induced investment incentives. We model this by introducing a public signal $y$, which is produced by the accounting information system of the firm. We assume that on date-1 both the realized cash flows $\left(z_{1}\right)$ and the accounting signal $(y)$ are publicly observable. The 
general idea is that $y$ may provide additional information beyond an aggregate cash flow report $\left(z_{1}\right)$ and in particular, $y$ may help differentiate the individual components of cash flows. ${ }^{7}$

\subsection{Input-based and Output-based Accounting Measures}

In accounting practice, two broad accounting measurement bases dominate how accounting deferrals/accruals are prepared. First, under an input-based measurement basis, accounting metrics are prepared to be estimates of the effort (or costs) expended in various firm activities. The historical (exchange) cost principle reflects this approach well. Ready examples are long-lived assets and inventory, where book values are based on acquisition costs. Second, under an output-based measurement basis, accounting metrics are prepared to be estimates of the expected reward in return (for the costly activities). The fair value principle reflects this approach well. Ready examples are market value methods where assets and liabilities are measured at market value or based on an estimate of the expected NPV of future cash flows (which is designed to simulate a would-be market value). We study both accounting systems and examine the effect of alternative accounting reports on the investment efficiency. We assume that the firm can choose either an input-based approach, labeled IP, or an output-based approach, labeled OP. ${ }^{8}$

Denote the signal produced by an output-based measure $y^{O P}$, and assume that

$$
y^{O P}=k \sqrt{\theta I}+\varepsilon^{O P}
$$

where $\varepsilon^{O P} \sim N\left(0, \sigma_{O P}^{2}\right)$. The output-based accounting report provides a noisy measurement of the short term investment return. ${ }^{9}$

For the input-based measure, denote the report $y^{I P}$, and assume that

$$
y^{I P}=I+\varepsilon^{I P}
$$

where $\varepsilon^{I P} \sim N\left(0, \sigma_{I P}^{2}\right)$. The input-based accounting report provides a noisy measurement of the investment cost. In the following, to simplify the notation, we denote the accounting policy by $m$, $m \in\{O P, I P\}$.

\footnotetext{
${ }^{7}$ This idea is consistent with some recognizable features of certain timing accruals. For example, the unearned revenue accruals help classify the timing properties of cash inflow, and the extraordinary-item category may help distinguish components of realized cash flows with different serial correlations.

${ }^{8}$ Here we have limited our attention to a single, one-time measurement. In practice, accounting measurement systems can be much more complex with an initial measurement, subsequent (date-2) re-valuation, and a final measurement on the disposal of the item in question. A dynamic model with multiple information arrivals would make it possible to model these issues, and it is certainly an interesting extension of the current model.

${ }^{9}$ For simplicity, we choose the short term return as the expected value of an output-based accounting report. Our results do not change if we assume that the report is scaled up to provide a noisy measurement of the total investment return (i.e., $y^{O P}=2 \sqrt{\theta I}+\varepsilon^{O P}$ ).
} 


\subsection{Equilibria under Alternative Accounting Regimes}

We now analyze the equilibrium behavior of the firm under both output-based accounting and input-based accounting.

Theorem 3 If $y^{O P}=k \sqrt{\theta I}+\varepsilon^{O P}$ and $y^{I P}=I+\varepsilon^{I P}$, using (6), there exists a unique linear equilibrium relative to $\Omega=\left\{z_{1}, y^{m}\right\}(m \in\{O P, I P\})$ and it is given by

(i) an equilibrium linear pricing function:

$$
\begin{aligned}
P\left(z_{1}, y^{m}\right) & =a^{m}+b_{z}^{m} \times z_{1}+b_{y}^{m} \times y^{m}, \text { where } \\
b_{z}^{O P} & =\frac{(1+\alpha) k^{2} \delta_{O P} \sigma^{2} \sigma_{\theta}^{2}+(1+\alpha) \sigma^{2} \sigma_{O P}^{2}+2 k \delta_{O P} \sigma_{O P}^{2} \sigma_{\theta}^{2}}{k^{2} \delta_{O P} \sigma^{2} \sigma_{\theta}^{2}+\sigma^{2} \sigma_{O P}^{2}+k^{2} \delta_{O P} \sigma_{O P}^{2} \sigma_{\theta}^{2}} \\
b_{y}^{O P} & =\frac{[2-(1+\alpha) k] k \delta_{O P} \sigma^{2} \sigma_{\theta}^{2}}{k^{2} \delta_{O P} \sigma^{2} \sigma_{\theta}^{2}+\sigma^{2} \sigma_{O P}^{2}+k^{2} \delta_{O P} \sigma_{O P}^{2} \sigma_{\theta}^{2}} \\
a^{O P} & =\left(2-b_{z}^{O P}\right) \mu+\left(2-k b_{z}^{O P}-k b_{y}^{O P}\right) \sqrt{\delta_{O P}} \theta_{0} \\
b_{z}^{I P} & =\frac{(1+\alpha) \delta_{I P}^{2} \sigma^{2} \sigma_{\theta}^{2}+(1+\alpha) \sigma^{2} \sigma_{I P}^{2}+2 k \delta_{I P} \sigma_{I P}^{2} \sigma_{\theta}^{2}}{\delta_{I P}^{2} \sigma^{2} \sigma_{\theta}^{2}+\sigma^{2} \sigma_{I P}^{2}+k^{2} \delta_{I P} \sigma_{I P}^{2} \sigma_{\theta}^{2}} \\
b_{y}^{I P} & =\frac{[2-(1+\alpha) k] \delta_{I P}^{\frac{3}{2}} \sigma^{2} \sigma_{\theta}^{2}}{\delta_{I P}^{2} \sigma^{2} \sigma_{\theta}^{2}+\sigma^{2} \sigma_{I P}^{2}+k^{2} \delta_{I P} \sigma_{I P}^{2} \sigma_{\theta}^{2}} \\
a^{I P} & =\left(2-b_{z}^{I P}\right) \mu+\left(2-k b_{z}^{I P}-\sqrt{\delta_{I P}} b_{y}^{I P}\right) \sqrt{\delta_{I P}} \theta_{0}
\end{aligned}
$$

(ii) an equilibrium investment function:

$$
\begin{aligned}
& I^{m}(\theta)=\left\{\begin{array}{ll}
\delta_{m} \theta, & \text { if } \theta \geq 0 \\
0 & \text { if } \theta<0
\end{array}\right. \text {, where } \\
& \delta_{O P}=\left(1-\beta+\frac{\beta k\left(b_{z}^{O P}+b_{y}^{O P}\right)}{2}\right)^{2}, \quad \delta_{I P}=\left(\frac{1-\beta+\frac{\beta k b_{z}^{I P}}{2}}{1-\beta b_{y}^{I P}}\right)^{2}
\end{aligned}
$$

To gain some insights into the results, we make the following observations.

- If $k=k^{*}=\frac{2}{1+\alpha}$, the linear equilibrium under either accrual accounting system produces the first-best investment level $\left(\delta_{m}=1\right)$ and the market response coefficients $b_{z}^{m}=\frac{2}{k}, b_{y}^{m}=0$ $(m \in\{O P, I P\})$. In this case, the cash flow $z_{1}$ provides sufficient information for efficient pricing, and the market ignores the accruals completely $\left(b_{y}^{m}=0\right)$.

- If $\sigma_{m}^{2} \rightarrow+\infty$, the equilibrium is the same as the basic setup $\left(\Omega=\left\{z_{1}\right\}\right)$. The quality of accruals is so poor that the market ignores the accounting signals $\left(b_{y}^{m}=0\right)$, which is equivalent to a setting without accounting reports. 
- The direction of the response to accounting signals (i.e., the sign of $b_{y}^{O P}$ or $b_{y}^{I P}$ ), can be positive or negative depending on the sign of $[2-(1+\alpha) k]$.

The following Corollary summarizes the intuitive properties of the equilibrium under either accounting regime.

Corollary 1 If $y^{O P}=k \sqrt{\theta I}+\varepsilon^{O P}$ and $y^{I P}=I+\varepsilon^{I P}$, using (6), for any $m \in\{O P, I P\}$,

(i) if $\sigma_{m}^{2} \rightarrow+\infty$, the investment choice approaches that in the basic setup where $\Omega=\left\{z_{1}\right\}$;

(ii) $\delta_{m}$ increases (decreases) in $\sigma_{m}^{2}$ when $k>k^{*}\left(k<k^{*}\right)$.

(iii) $b_{z}^{m}$ decreases (increases) in $\sigma_{m}^{2}$ when $k>k^{*}\left(k<k^{*}\right)$.

(iv) $b_{y}^{m}$ increases (decreases) in $\sigma_{m}^{2}$ when $k>k^{*}\left(k<k^{*}\right)$.

This corollary confirms an intuitive relation between measurement noise and investment efficiency. Recall that Theorem 2 shows that the aggregation of on-going and investment cash flows induces the sub-optimal investment. The combination of items (i) and (ii) of Corollary 1 indicates that the sub-optimal investment problem is alleviated by the accounting report. For example, when $k<k^{*}$, and $\sigma_{m}^{2} \rightarrow+\infty$, we know $\delta_{m}$ is the same as in the basic setup and the firm underinvests. In this case, item (ii) implies that a lower $\sigma_{m}^{2}($ than $+\infty)$ would induce a higher $\delta_{m}$, alleviating the under-investment problem.

When the accounting quality is extremely poor $\left(\sigma_{m}^{2} \rightarrow+\infty\right)$, no valuation weight is placed on the accounting signals $\left(b_{y}^{m}=0\right)$. As the quality improves, more weight is shifted between the cash flow report and the accounting report. For example, when $k<k^{*}$, the market under-prices the investment. As $\sigma_{m}^{2}$ is lowered (from $+\infty$ ), the market response to the accounting signal increases (from zero) and the response to the cash report decreases (i.e., the weight shifts from cash flow to accruals).

Overall, the results thus far show that, when the noise level is high enough, an improvement in the quality of the accounting measures (i.e., a drop in the noise) improves the communication between the firm and the market, and this benefits the investment efficiency. That is, the quality of accruals is well-defined and well-behaved: the lower the variance, the higher the accrual quality, the lower the market mispricing of firm investments, and most importantly, the more efficient the investment decision.

\subsection{Comparing Output-based and Input-based Accounting Measures}

When the noise level is low, the output-based and input-based measures exhibit a fundamental difference. We find a monotonic relation between the investment efficiency and the quality of the output-based accounting signal. That is, the investment efficiency under output-based accounting continues to improve when measurement noise decreases.

In the extreme, output-based accounting achieves the first-best result as $\sigma_{O P}^{2}$ is reduced to zero. If $\sigma_{O P}^{2}=0$, the market is able to infer the short-term investment return perfectly from 
the accounting report (because $y_{O P}=k \sqrt{\theta I}$ for certain). Subtracting the accounting signal from the aggregate cash flow reveals the cash flow from first period on-going activities. That is, the accounting report helps the investors clearly distinguish the cash flow components. In turn, the market response coefficients are $b_{z}^{O P}=1+\alpha$ and $b_{y}^{O P}=\frac{2}{k}-(1+\alpha)$, leading to a combined reaction of $\frac{2}{k}$ to the short-term investment return, which provides the first-best investment incentive.

In contrast, input-based accounting is another story in which the results are not as straightforward. The relation between investment efficiency and measurement noise $\left(\sigma_{I P}^{2}\right)$ is not monotonic. When $k>k^{*}$, the over-investment problem exists only when $\sigma_{I P}^{2}$ is high enough. If $\sigma_{I P}^{2}$ is very low, the firm underinvests. In the extreme, when $\sigma_{I P}^{2}=0$, outsiders are able to infer the actual investment made $(I)$; the market response coefficients are $b_{z}^{I P}=1+\alpha$, and $b_{y}^{I P}=[2-(1+\alpha) k] \delta_{I P}^{-\frac{1}{2}}$, and the investment level $\delta_{I P}=\left(1-\beta\left(\frac{k(1+\alpha)}{2}-1\right)\right)^{2}<1$.

The following theorem summarizes and compares the effects of the two accounting systems on the investment efficiency.

Theorem 4 If $y^{O P}=k \sqrt{\theta I}+\varepsilon^{O P}$ and $y^{I P}=I+\varepsilon^{I P}$,

(i) if $\sigma_{O P}^{2}=0$, investment choice is the first-best level, and

(ii) if $\sigma_{I P}^{2}=\sigma_{\theta}^{2}$, investment choice is the first-best level.

(iii) There exists a $\Sigma\left(0<\Sigma<\sigma_{\theta}^{2}\right)$, such that a sufficient condition for input-based accounting to be more (less) efficient than the cash flow reporting regime (of the basic setup) is $\sigma_{I P}^{2}>\Sigma$ $\left(\sigma_{I P}^{2}<\Sigma\right)$.

(iv) There exists a $\Sigma^{\prime}\left(\Sigma<\Sigma^{\prime}<\sigma_{\theta}^{2}\right)$, such that a sufficient condition for input-based accounting to be more efficient than output-based accounting is the combination of (a) $\sigma_{I P}^{b} \in\left[\Sigma^{\prime}, \sigma_{\theta}^{2}\right]$ and (b) $\sigma_{O P}^{2}>\Sigma^{\prime}$.

The two systems achieve the first-best at different noise levels. Under output-based, the firstbest is achieved when the accounting measure is noiseless, which is very intuitive. Under the inputbased system, the first-best is achieved when the noise is small but not zero. More importantly, output-based does not dominate input-based in all situations. Since some noise is unavoidable in practice, it is likely input-based may be preferable. Consider the following two comparisons. First, suppose an accounting item in question is well-understood and easy to measure. So we assume both output-based and input-based measures share the same (small) noise level (e.g., less than $\sigma_{\theta}^{2}$ ). In this case, Theorem 4 predicts input-based is preferred if the variance of the noise $\left(\sigma_{m}^{2}\right)$ is between $\Sigma^{\prime}$ and $\sigma_{\theta}^{2}$. Alternatively, suppose the accounting item is not well-understood and hard to measure. So we assume the noise level is high for both measures (i.e., greater than $\sigma_{\theta}^{2}$ ). In this case, it is more likely that output-based accounting may entail a more noisy measure than input-based. ${ }^{10}$ As a result, the investment efficiency under a highly noisy output-based measure may be closer to

\footnotetext{
${ }^{10}$ One way to view the natural relation between $\sigma_{I P}^{2}$ and $\sigma_{O P}^{2}$ is that the accounting system constructs the outputbased measure based on two estimates: an estimate of actual investment made $\left(\mathrm{e} \cdot \mathrm{g}, y^{I P}=\hat{I}=I+n o i s e\right)$ and an estimate of profitability (e.g., $\hat{\theta}=\theta+$ noise). The output-based measure is estimated using the true production
} 
the (benchmark) cash flow setting (by Corollary 1) while the efficiency under a not-so-noisy inputbased measure may be closer to first-best (by Theorem 4). In this case, input-based dominates output-based as long as their variance difference is large enough.

The key difference between input-based and output-based has to do with the fundamental difference between the two accounting approaches. The input-based method $\left(y^{I P}=I+\varepsilon^{I P}\right)$ requires estimating the investment cost $(I)$ alone, without any explicit attention to the profitability of the investment $(\theta)$. The output-based method $\left(y^{O P}=k \sqrt{\theta I}+\varepsilon^{O P}\right)$ requires estimating both $I$ and $\theta$. This fundamental difference leads to a structural difference in the market mispricing of firm investments.

Returning to the analysis of investment distortion induced by mispricing, substitute the pricing function $\left(P=a^{O P}+b_{z}^{O P}\left(x_{1}+k \sqrt{\theta I}\right)+b_{y}^{O P}\left(k \sqrt{\theta I}+\varepsilon^{O P}\right)\right)$ into the expected mispricing, and we have

$$
\begin{aligned}
& E_{x_{1} x_{2}}\left[P-\left(x_{1}+x_{2}+2 \sqrt{\theta I}\right) \mid \theta\right] \\
& =E_{x_{1} x_{2}}\left[a^{O P}+b_{z}^{O P}\left(x_{1}+k \sqrt{\theta I}\right)+b_{y}^{O P}\left(k \sqrt{\theta I}+\varepsilon^{O P}\right)-\left(x_{1}+x_{2}+2 \sqrt{\theta I}\right) \mid \theta\right] \\
& =\left[\left(b_{z}^{O P}+b_{y}^{O P}\right) k-2\right] \sqrt{\theta I}+E_{x_{1} x_{2}}\left[a^{O P}+b_{z}^{O P} x_{1}+b_{y}^{O P} \varepsilon^{O P}-\left(x_{1}+x_{2}\right)\right]
\end{aligned}
$$

If $\left(b_{z}^{O P}+b_{y}^{O P}\right) k-2 \neq 0$, any investment will affect the market pricing, giving the firm an incentive to over- or under-invest. The marginal effect of investment $I$ on the mispricing depends on true investment profitability theta (because the derivative equals $\left.\frac{\left(b_{z}^{O P}+b_{y}^{O P}\right) k-2}{2} \sqrt{\frac{\theta}{I}}\right)$. This leads to a dampening effect: the marginal benefit is concave in $I$, providing a diminishing return to investment deviations. Intuitively, the firm's ability to use real investment to change the market perception of its investment profitability is mitigated by the independent profitability estimate built into the output-based measure.

With input-based accounting, the expected mispricing is

$$
\begin{aligned}
& E_{x_{1} x_{2}}\left[P-\left(x_{1}+x_{2}+2 \sqrt{\theta I}\right) \mid \theta\right] \\
& =E_{x_{1} x_{2}}\left[a^{I P}+b_{z}^{I P}\left(x_{1}+k \sqrt{\theta I}\right)+b_{y}^{I P}\left(I+\varepsilon^{I P}\right)-\left(x_{1}+x_{2}+2 \sqrt{\theta I}\right) \mid \theta\right] \\
& =\left(b_{z}^{I P} k-2\right) \sqrt{\theta I}+b_{y}^{I P} I+E_{x_{1} x_{2}}\left[a^{I P}+b_{z}^{I P} x_{1}+b_{y}^{I P} \varepsilon^{I P}-\left(x_{1}+x_{2}\right)\right]
\end{aligned}
$$

The mispricing will only affect the equilibrium investment through the first two terms: $\left(b_{z}^{I P} k-\right.$ 2) $\sqrt{\theta I}+b_{y}^{I P} I$. The marginal effect of investment on the first term $\left(b_{z}^{I P} k-2\right) \sqrt{\theta I}$ depends on the investment profitability (the derivative is $\frac{b_{z}^{I P} k-2}{2} \sqrt{\frac{\theta}{I}}$ ) while the marginal effect on the second term $b_{y}^{I P} I$ depends only on an equilibrium constant $b_{y}^{I P}$. The dampening effect is active only on the first term, not the second term. Notice, from Theorem 3, that we know in equilibrium, the market

function (roughly, $y^{O P}=2 \sqrt{\hat{\theta} \hat{I}}$ ). Viewed this way, it is natural that the overal noise in $y^{O P}$ is likely to be higher than the noise in $y^{I P}$. 
reactions to the cash flow and accounting measures $\left(b_{z}^{I P}\right.$ and $b_{y}^{I P}$ respectively) are such that

$$
\begin{aligned}
b_{z}^{I P} k-2 & =\frac{[(1+\alpha) k-2]\left(\sigma^{2} \sigma_{I P}^{2}+\delta_{I P}^{2} \sigma^{2} \sigma_{\theta}^{2}\right)}{\delta_{I P}^{2} \sigma^{2} \sigma_{\theta}^{2}+\sigma^{2} \sigma_{I P}^{2}+k^{2} \delta_{I P} \sigma_{I P}^{2} \sigma_{\theta}^{2}} \\
b_{y}^{I P} & =\frac{[2-(1+\alpha) k] \delta_{I P}^{\frac{3}{2}} \sigma^{2} \sigma_{\theta}^{2}}{\delta_{I P}^{2} \sigma^{2} \sigma_{\theta}^{2}+\sigma^{2} \sigma_{I P}^{2}+k^{2} \delta_{I P} \sigma_{I P}^{2} \sigma_{\theta}^{2}} .
\end{aligned}
$$

When $k \neq k^{*}$, the sign of $b_{z}^{I P} k-2$ is always the opposite of the sign of $b_{y}^{I P}$, which indicates that the marginal effects on the two terms are in the opposite direction. The total effects on mispricing depend on which item outweighs the other.

For example, if $k>k^{*}, b_{z}^{I P} k-2$ is positive which indicates that a higher investment would increase market mispricing. However, the dampening effect provides a diminishing return to overinvestment which makes over-investment less attractive. On the other hand, the firm is also motivated to under-invest because $b_{y}^{I P}$ is negative. Notice here that the marginal effect is a constant and is independent of the private information $\theta$ and investment level $I$; no dampening is in effect. This hurts the economy when the accounting report is too precise. If $\sigma_{I P}^{2}$ is too small, the absolute value of $b_{y}^{I P}$ is too large, which motivates the firm to under-invest by a large amount (Corollary 1 ). This motivation outweighs the over-investment motivation by the first item because a small $\sigma_{I P}^{2}$ reduces the market response to the aggregate cash flows report $\left(b_{z}^{I P}\right)$.

Consider the limiting case, when $\sigma_{I P}^{2}=0$. Unlike the case for output-based accounting, the first-best investment is not achieved when the input-based measure is noiseless. Suppose the firm invests the first-best amount (e.g., $I=\theta$ ), then the market's best responses are $b_{z}^{I P}=1+\alpha$ and $b_{y}^{I P}=2-(1+\alpha) k<0$. These responses invite the firm to under-invest because at $I=\theta$, the marginal benefit of additional investment is $\frac{b_{z}^{I P} k-2}{2}+b_{y}^{I P}=1-\frac{(1+\alpha) k}{2}$, which is less than the marginal cost of additional investment $(=1) \cdot{ }^{11}$

In another knife-edge case, the two opposite effects exactly offset each other where the first-best is achieved. That is, if $\sigma_{I P}^{2}=\sigma_{\theta}^{2}$ and we propose that $\delta_{I P}=1$, then we find that $\frac{b_{z}^{I P} k-2}{2}+$ $b_{y}^{I P}=0$ (i.e., the marginal effect of any investment deviation is zero). In equilibrium, there is no incentive to distort investment. Thus, with the input-based measure, the first-best is achieved when measurement noise is small but not zero. Table 1 summarizes the performance of three accounting

\footnotetext{
${ }^{11}$ Further, in this limiting case of perfect knowledge of the actual investment made $(I)$, the induced investment efficiency is worse than that of the basic setup (item $i v$ of the Theorem 4). Alternatively, if the profitability $(\theta)$ is perfectly revealed and $z$ is reported, it can be shown that the induced efficiency is not first-best. This is consistent with Kanodia et al (2005) where some imprecision in the accounting measurement is preferred. What is different in the current model is that the conclusion on accounting imprecision depends on the accounting measurement basis. With an output-based measure, the ideal accounting is noiseless.
} 
regimes

Table1: The performance of three accounting regimes

\begin{tabular}{|c|c|c|c|c|c|c|}
\hline & \multicolumn{6}{|c|}{ Parameter Regions } \\
\hline \multirow{2}{*}{$\begin{array}{c}\text { Accounting } \\
\text { Regimes }\end{array}$} & \multirow[t]{2}{*}{$\beta=0$} & \multicolumn{5}{|c|}{$0<\beta<1$} \\
\hline & & $k=k^{*} \equiv \frac{2}{1+\alpha}$ & \multicolumn{2}{|c|}{$k>k^{*}$} & \multicolumn{2}{|c|}{$k<k^{*}$} \\
\hline Cash Flow & $\delta=1$ & $\delta=1$ & \multicolumn{2}{|c|}{$\delta>1$} & \multicolumn{2}{|c|}{$\delta<1$} \\
\hline Output-based & $\delta_{O P}=1$ & $\delta_{O P}=1$ & $\begin{array}{l}\sigma_{O P}^{2}=0 \\
\delta_{O P}=1\end{array}$ & $\begin{array}{l}\sigma_{O P}^{2}>0 \\
\delta_{O P}>1\end{array}$ & $\begin{array}{l}\sigma_{O P}^{2}=0 \\
\delta_{O P}=1\end{array}$ & $\begin{array}{l}\sigma_{O P}^{2}>0 \\
\delta_{O P}<1\end{array}$ \\
\hline Input-based & $\delta_{I P}=1$ & $\delta_{I P}=1$ & $\begin{array}{c}\sigma_{I P}^{2}=\sigma_{\theta}^{2} \\
\delta_{I P}=1\end{array}$ & $\begin{array}{c}\sigma_{I P}^{2} \lessgtr \sigma_{\theta}^{2} \\
\delta_{I P} \lessgtr 1\end{array}$ & $\begin{array}{c}\sigma_{I P}^{2}=\sigma_{\theta}^{2} \\
\delta_{I P}=1\end{array}$ & $\begin{array}{c}\sigma_{I P}^{2} \lessgtr \sigma_{\theta}^{2} \\
\delta_{I P} \gtrless 1\end{array}$ \\
\hline
\end{tabular}

Figure 2 illustrates the effect of accounting reports on the efficiency. Here, the expected net project return represents the efficiency of the investment. The FB line stands for the net project return when the investment level is the first-best $\left(I^{F B}=\theta\right)$. The basic setting results $\left(I^{S B}=\delta \theta\right)$ are denoted by the SB-CF line. The performance of input-based accounting $\left(I^{I P}=\delta_{I P} \theta\right)$ and outputbased accounting $\left(I^{O P}=\delta_{O P} \theta\right)$ is described by the IP and OP solid curves respectively. From the figure, it is easy to see that output-based accounting is dominated by input-based accounting in the region when the common noise is between $\Sigma^{\prime}$ and $\sigma_{\theta}^{2} \cdot{ }^{12}$

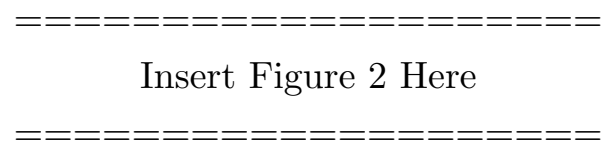

\section{Extension I: Accounting Manipulation}

In this section we expand the model to consider managerial manipulation of the accounting measurement. A robust feature of any accrual measurement is that firms have varying degrees of influence (or discretion) on how accruals are prepared. However, other economic factors (e.g., auditing or managerial reputation) prevent the use of complete discretion. We capture this partial discretion by considering a simple model of cost-benefit calculus on the part of the firm.

\footnotetext{
${ }^{12}$ The Figure 2 shows that input-based accounting induces more efficient investment decisions than output-based accounting for all noise level greater than $\Sigma^{\prime}$. We note that this result is not general and that there exist sufficient conditions that the output-based accounting dominates the input-based accounting when the measurement noise is large. That is, when the noise is high, input-based does not necessarily dominate output-based when both share a common measurement noise.
} 


\subsection{Equilibrium under Accounting Manipulation}

Suppose that the accounting signal $y^{m}(m \in\{O P, I P\})$ is subjected to managerial manipulation. A firm can prepare its accounting report $w^{m}$ differently from the unmanipulated $y^{m}$, at a cost. We assume the cost as $c\left(w^{m}\right)=\frac{c^{m}}{2}\left(w^{m}-y^{m}-\xi^{m}\right)^{2}$, where $\xi^{m}$ is independent of all other random variables and follows a normal distribution with mean zero and variance of $\eta_{m}^{2}$. Variable $\xi^{m}$ captures the random component of manipulation costs. ${ }^{13}$ From the earlier results, the market response to the accounting report can be positive or negative. Then, with accounting manipulation, the firm can benefit from adjusting the accounting report upwards or downwards, at the margin. We next introduce the definition of an equilibrium for the setup with accounting manipulation.

Definition 2 An equilibrium relative to $\Omega=\left\{z_{1}, w^{m}\right\}$ consists of an investment function $I_{w}^{m}(\cdot)$, a reporting policy $w^{m}(\cdot)$, and a market pricing function $P(\cdot)$, such that:

(i) Given $P(\cdot)$, the optimal investment function $I_{w}^{m}(\cdot)$ and the reporting policy $w^{m}(\cdot)$ maximize $V\left(\theta \mid I_{w}^{m}(\cdot), w^{m}(\cdot)\right)=E\left[-I_{w}^{m}+\beta P(\cdot)+(1-\beta)\left(z_{1}+z_{2}\right)-c\left(w^{m}\right)\right]$

(ii) Given $I_{w}^{m}(\cdot)$ and $w^{m}(\cdot)$, the pricing function $P(\cdot)$ satisfies $P=E\left[z_{1}+z_{2} \mid \Omega, I_{w}^{m}(\cdot), w^{m}(\cdot)\right]$

We now analyze the equilibrium behavior of the firm under the accrual basis accounting with manipulation.

Theorem 5 If $y^{O P}=k \sqrt{\theta I}+\varepsilon^{O P}$ and $y^{I P}=I+\varepsilon^{I P}$, and $c\left(w^{m}\right)=\frac{c^{m}}{2}\left(w^{m}-y^{m}-\xi^{m}\right)^{2}(m \in$ $\{O P, I P\})$, where $k \in(0,2)$ and using (6), there exists a unique linear equilibrium relative to $\Omega=\left\{z_{1}, w^{m}\right\}$. It is given by

(i) an equilibrium linear pricing function:

$$
\begin{gathered}
P\left(z_{1}, w^{m}\right)=a_{w}^{m}+b^{m} \times z_{1}+d^{m} \times w^{m}, \text { where } \\
b^{O P}=\frac{(1+\alpha) k^{2} \gamma_{O P} \sigma^{2} \sigma_{\theta}^{2}+(1+\alpha) \sigma^{2} v_{O P}^{2}+2 k \gamma_{O P} v_{O P}^{2} \sigma_{\theta}^{2}}{k^{2} \gamma_{O P} \sigma^{2} \sigma_{\theta}^{2}+\sigma^{2} v_{O P}^{2}+k^{2} \gamma_{O P} v_{O P}^{2} \sigma_{\theta}^{2}} \\
d^{O P}=\frac{[2-(1+\alpha) k] k \gamma_{O P} \sigma^{2} \sigma_{\theta}^{2}}{k^{2} \gamma_{O P} \sigma^{2} \sigma_{\theta}^{2}+\sigma^{2} v_{O P}^{2}+k^{2} \gamma_{O P} v_{O P}^{2} \sigma_{\theta}^{2}} \\
a_{w}^{O P}=\left(2-b^{O P}\right) \mu+\left(2-k b^{O P}-k d^{O P}\right) \sqrt{\gamma_{O P}} \theta_{0}-\frac{\beta\left(b^{O P}\right)^{2}}{c^{O P}}
\end{gathered}
$$

\footnotetext{
${ }^{13}$ See a similar assumption and more discussions of the cost structure in Dye and Sridhar 2004a.
} 


$$
\begin{aligned}
b^{I P} & =\frac{(1+\alpha) \gamma_{I P}^{2} \sigma^{2} \sigma_{\theta}^{2}+(1+\alpha) \sigma^{2} v_{I P}^{2}+2 k \gamma_{I P} v_{I P}^{2} \sigma_{\theta}^{2}}{\gamma_{I P}^{2} \sigma^{2} \sigma_{\theta}^{2}+\sigma^{2} v_{I P}^{2}+k^{2} \gamma_{I P} v_{I P}^{2} \sigma_{\theta}^{2}} \\
d^{I P} & =\frac{[2-(1+\alpha) k] \gamma_{I P}^{\frac{3}{2}} \sigma^{2} \sigma_{\theta}^{2}}{\gamma_{I P}^{2} \sigma^{2} \sigma_{\theta}^{2}+\sigma^{2} v_{I P}^{2}+k^{2} \gamma_{I P} v_{I P}^{2} \sigma_{\theta}^{2}} \\
a_{w}^{I P} & =\left(2-b^{I P}\right) \mu+\left(2-k b^{I P}-\sqrt{\gamma_{I P}} d^{I P}\right) \sqrt{\gamma_{I P}} \theta_{0}-\frac{\beta\left(d^{I P}\right)^{2}}{c^{I P}} \\
v_{m}^{2} & =\sigma_{m}^{2}+\eta_{m}^{2}
\end{aligned}
$$

(ii) an equilibrium investment function and an equilibrium reporting policy:

$$
\begin{aligned}
I_{w}^{m}(\theta) & =\left\{\begin{array}{cc}
\gamma_{m} \theta, & \text { if } \theta \geq 0 \\
0 & \text { if } \theta<0
\end{array}\right. \text { where } \\
\gamma_{O P} & =\left(1-\beta+\frac{\beta k\left(b^{O P}+d^{O P}\right)}{2}\right)^{2}, \quad \gamma_{I P}=\left(\frac{1-\beta+\frac{\beta k b^{I P}}{2}}{1-\beta d^{I P}}\right)^{2} \\
w^{m} & =y^{m}+\xi^{m}+\frac{\beta d^{m}}{c^{m}}
\end{aligned}
$$

According to the equilibrium reporting policy (the $w^{m}$ expression in equation 26), the equilibrium accounting report varies from the one without accounting manipulation: the additional noise from a random variable $\xi^{m}$ is added as well as a fixed constant $\frac{\beta d^{m}}{c^{m}}$.

The market can perfectly calculate the fixed constant $\frac{\beta d^{m}}{c^{m}}$. (Parameters $\beta$ and $c^{m}$ are common knowledge, and $d^{m}$ is the equilibrium market response to the accounting report.) The intercept of the pricing function $a_{w}^{m}$ is adjusted accordingly to "undo" the expected manipulation. The random component of manipulation injects noise $\xi^{m}$ into the accounting report. As a result, accounting manipulation worsens the quality of accounting reports. The pricing function is adjusted in a way that the variance of the measurement noise $\left(\sigma_{m}^{2}\right)$ in the previous equilibrium pricing function is replaced by $v_{m}^{2}$, which equals to the sum of $\sigma_{m}^{2}$ and $\eta_{m}^{2}$.

\subsection{Value of Accounting Manipulation}

The accounting manipulation leads to a more noisy accounting measure and a dead-weight loss (the manipulation cost to the firm). The latter cost is incorporated into the following cost-benefit analysis of accounting manipulation.

Corollary 2 Under output-based, accounting manipulation always makes the firm worse off; under input-based accounting, a sufficient condition for accounting manipulation to be value-enhancing is the combination of (i) $v_{I P}^{2}<\sigma_{\theta}^{2}$ and (ii) $c^{I P} \cdot \theta_{0}$ is sufficiently high. 
As we have shown, the performance of output-based accounting monotonically decreases as the quality of the accounting report worsens. Therefore, under output-based, accounting manipulation reduces efficiency for two reasons. First, a more noisy accounting report induces less efficient investment. Second, the firm incurs a manipulation $\operatorname{cost} c\left(w^{m}\right)$.

However, with input-based accounting, the non-monotonicity feature makes it possible that the incremental noise due to manipulation may benefit the firm. Recall that in Figure 2, making the accruals more noisy can improve efficiency when the accruals are "too" precise $\left(\sigma_{I P}^{2}<\sigma_{\theta}^{2}\right)$. Thus, if $v_{I P}^{2}<\sigma_{\theta}^{2}$, the expected net project return strictly increases as a result of accounting manipulation, provided that $\eta_{I P}^{2}$ is not too large.

Since the expected net project return is $\left(2 \sqrt{\gamma_{m}}-\gamma_{m}\right) \cdot \theta_{0}$, the higher $\theta_{0}$ can magnify the gain from more efficient investment choices. The cost of manipulation is

$$
c\left(w^{I P}\right)=\frac{c^{I P}}{2}\left(w^{I P}-y^{I P}-\xi^{I P}\right)^{2}=\frac{\left(\beta d^{I P}\right)^{2}}{2 c^{I P}}
$$

A higher $c^{I P}$ can reduce the dead-weight loss from the firm's myopic decision. Thus, if $c^{I P} \cdot \theta_{0}$ is sufficiently high, the cost of earnings management is outweighed by the increase of the expected investment returns. ${ }^{14}$

Intuitively, when the actual investment made is measured too precisely, the market pricing places too much valuation weight on the accounting report, providing an unmitigated incentive to over- or under-invest. By allowing accounting manipulation, more noise is injected into accounting reports and the market reacts by reducing the valuation weight. As a result, less pressure leads to

\footnotetext{
${ }^{14}$ It might also be interesting to compare the efficiency of discretionary accounting reports with the basic cash flow setting. This takes the view that allowing either output-based or input-based accounting measurement implicitly grants the firm the ability to manipulate their accounting performance. In other words, accrual accounting and the manipulation option are a bundle.

From Theorem 4, under output-based accounting, the investment is always more efficient than in the cash flow setup, but manipulation always incurs positive $\operatorname{costs} c\left(w^{O P}\right)$. Whether output-based accounting with manipulation is preferable to the cash flow setup depends on the cost incurred and on benefits derived from the investment improvement. The same tension exists under input-based cost accounting with manipulation. Specifically, inputbased accounting with manipulation is more efficient than the cash flow setup when (i) $v_{I P}^{2}>\Sigma$ and (ii) $c \cdot \theta_{0}$ is sufficiently high. On the other hand, input-based accounting with manipulation is less efficient than the cash flow setup when $v_{I P}^{2}<\Sigma$.
} 
less inefficient investment choices. ${ }^{15}$ Table 2 summarizes the effect of accounting manipulation.

Table 2: The effect of accounting manipulation for different accounting regimes

\begin{tabular}{|c|c|c|c|}
\hline & \multicolumn{2}{|c|}{ Parameter Region } \\
\hline \multicolumn{2}{|c|}{ Accounting Regimes } & $k>k^{*}$ & $k<k^{*}$ \\
\hline \multicolumn{2}{|c|}{ Output-based } & $\gamma_{O P}>1, \frac{\partial}{\partial v_{O P}^{2}} \gamma_{O P}>0$ & $\gamma_{O P}<1, \frac{\partial}{\partial v_{O P}^{2}} \gamma_{O P}<0$ \\
\hline \multirow{2}{*}{ Input-based } & $\frac{v_{I P}^{2}>\sigma_{\theta}^{2}}{v_{I P}^{2}<\sigma_{\theta}^{2}}$ & $\frac{\gamma_{I P}>1, \frac{\partial}{\partial v_{I P}^{2}} \gamma_{I P}>0}{\gamma_{I P}<1, \frac{\partial}{\partial v_{I P}^{2}} \gamma_{I P}<0}$ & $\frac{\gamma_{I P}<1, \frac{\partial}{\partial v_{I P}^{2}} \gamma_{I P}<0}{\gamma_{I P}>1, \frac{\partial}{\partial v_{I P}^{2}} \gamma_{I P}>0}$ \\
\hline
\end{tabular}

\section{3 "Real" versus "Accounting" Manipulation}

The above analysis points to a link between the so-called "real" earnings management and "accounting" earnings management. Real management typically refers to the firm's discretionary choices which affect the firm's cash flow for the sole purpose of inflating reported performance. These choices are not in the best interest of the shareholders. Accounting management typically refers to the firm's discretionary choices which affect the firm's reported performance by altering the accounting measurement process. In our model, we interpret investment deviations from the first-best as an example of real earnings management and accounting manipulation of $y$ into $w$ as accounting earnings management.

Under the output-based measure, our results indicate that accounting earnings management always leads to more real management. They are complements. This is because "accounting" management leads to more mispricing. Under an input-based measure, a similar result is obtained when the measurement noise is high. However, when noise is low, (especially in the region where investment efficiency increases in noise,) accounting earnings management leads to less mispricing, and more efficient investment choices are made. Here accounting management is a substitute for real management. The intuition is that the accounting management introduces additional noise, which leads to less market reaction to the accounting report. Less pressure on the accounting numbers mitigates over- and under-investment incentives.

\section{Extension II: Technology for future investments}

In this section we modify the model to include a situation where the firm technology may be re-used in the future. That is, from date- 2 onwards, the owners of the firm may generate future cash flow by investing in the firm technology they have acquired on date-1. In other words, the owners have an option to invest and will exercise the option if the technology turns out to be profitable. In turn,

\footnotetext{
${ }^{15}$ This intuition does not follow when output-based is used because when the measurement noise is low, actual investment only partially affects the accounting signal (recall $y^{O P}=k \sqrt{\theta I}+\varepsilon^{O P}$ ).
} 
on date-1, the capital market not only prices the cash flows generated from the date- 0 investment, it also prices the value of owning the technology that produces future cash flows. ${ }^{16}$

\subsection{Model Modifications}

To simplify the problem, we change the model as the following. On date- 0 , the firm chooses an investment level, denoted $I_{1} \in \mathbb{R}^{+}$, based on the private signal $\theta$, same as before.

On date-1, shares of the firm are traded in a competitive capital market. However, we assume there are no on-going activities ${ }^{17}\left(x_{1}\right.$ and $\left.x_{2}\right)$ and only one public signal $\lambda^{m}(m \in\{O P, I P\})$, which is produced by the accounting information system, is observable to outsiders. Similarly, we assume that the firm can choose either an input-based approach $(I P)$, or an output-based approach $(O P)$ as the accounting measurement bases. For the output-based measure,

$$
\lambda^{O P}=2 \sqrt{\theta I_{1}}+\varepsilon^{O P}
$$

where $\varepsilon^{O P} \sim N\left(0, \sigma_{O P}^{2}\right)$. The output-based accounting reports provide a noisy measurement of the total investment return. For input-based measure,

$$
\lambda^{I P}=I_{1}+\varepsilon^{I P}
$$

where $\varepsilon^{I P} \sim N\left(0, \sigma_{I P}^{2}\right)$. The input-based accounting reports provide a noisy measurement of the investment costs.

Finally, on date- 2 , the owners observe the return of the initial investment $2 \sqrt{\theta I_{1}}$, and choose additional investments into the existing technology. We assume the present value of future cashflows is $r \theta, r \in \mathbb{R}^{+}$. A simple interpretation of this representation is that the future profitability of the technology is the same as $\theta$, and the future owners make optimal investment decision, knowing the true $\theta$. In this case, in every period this technology is viable, the owners choose optimal $I_{t}$ to maximize $2 \sqrt{\theta I_{t}}-I_{t}$ and generate periodic profits equal to $\theta$, assuming a positive $\theta$. As a result, future profits can be represented by an annuity (or perpetuity). Parameter $r$ summarizes the importance of these future cashflows relative to the cashflow generated by the initial investment. If the technology is long-lived or if the owners' discount rate is low, more firm value comes from future investments (or "growth opportunities"), leading to a higher $r$.

A more complex, perhaps more realistic, interpretation involves a non-stationary technology, or future firm owners subjected to additional market frictions, or that the true $\theta$ is revealed to the owners gradually through learning-by-doing. However, under these scenarios, it may be reasonable to assume that the value of this re-investment option is proportional to the past profitability $\theta$. As

\footnotetext{
${ }^{16}$ We wish to thank the referee for suggesting us to pursue this extension.

${ }^{17}$ This assumption is made for simplicity only. All results in this section survive if a stochastic on-going activities are introduced as long as, as before, they are independent of other random variables in the model.
} 
a result, we believe our characterization is a reasonable approximation to capture the idea of this option without bringing in additional complexity to the model.

\subsection{Equilibrium under Modified Model}

Now return to the pricing problem on date-1, without the on-going cashflows, the capital market must estimate the value of the date- 2 cashflow generated by past investment and the value of the potential future cashflows generated by future investments. As a result, the market price is equal to the expected value of the cash flow from existing project plus the present value of the reinvestment option, that is

$$
P=E\left[2 \sqrt{\theta I_{1}}+r \theta \mid \Omega\right] .
$$

As before, when making the initial investment on date-0, the firm is motivated by both the long-term interest and the short term interest. The convex combination of these concerns is the same as the basic setup, that is $-I_{1}+\beta P+(1-\beta)\left(2 \sqrt{\theta I_{1}}+r \theta\right)$.

We now analyze the equilibrium behavior of the firm with technology for future investments.

Theorem 6 If $\lambda^{O P}=2 \sqrt{\theta I_{1}}+\varepsilon^{O P}$ and $\lambda^{I P}=I_{1}+\varepsilon^{I P}$, and using (6), there exists a unique linear equilibrium relative to $\Omega=\left\{\lambda^{m}\right\}$. It is given by

(i) an equilibrium linear pricing function:

$$
\begin{aligned}
P\left(\lambda^{m}\right) & =a_{\lambda}^{m}+b_{\lambda}^{m} \times \lambda^{m}, \text { where } \\
b_{\lambda}^{O P} & =\frac{\left(4 \gamma_{O P}^{\prime}+2 r \sqrt{\gamma_{O P}^{\prime}}\right) \sigma_{\theta}^{2}}{4 \gamma_{O P}^{\prime} \sigma_{\theta}^{2}+\sigma_{O P}^{2}} \\
a_{\lambda}^{O P} & =\left(2 \sqrt{\gamma_{O P}^{\prime}}+r-2 \sqrt{\gamma_{O P}^{\prime}} b_{\lambda}^{O P}\right) \theta_{0} \\
b_{\lambda}^{I P} & =\frac{\left(2 \sqrt{\gamma_{I P}^{\prime}}+r\right) \gamma_{I P}^{\prime} \sigma_{\theta}^{2}}{\left(\gamma_{I P}^{\prime}\right)^{2} \sigma_{\theta}^{2}+\sigma_{I P}^{2}} \\
a_{\lambda}^{I P} & =\left(2 \sqrt{\gamma_{I P}^{\prime}}+r-\gamma_{I P}^{\prime} b_{\lambda}^{I P}\right) \theta_{0}
\end{aligned}
$$

(ii) an equilibrium investment function:

$$
\begin{aligned}
I_{1}^{m}(\theta) & =\left\{\begin{array}{cc}
\gamma_{m}^{\prime} \theta, & \text { if } \theta \geq 0 \\
0 & \text { if } \theta<0
\end{array}\right. \text { where } \\
\gamma_{O P}^{\prime} & =\left(1-\beta+\beta b_{\lambda}^{O P}\right)^{2}, \quad \gamma_{I P}^{\prime}=\left(\frac{1-\beta}{1-\beta b_{\lambda}^{I P}}\right)^{2}
\end{aligned}
$$

With a valuable technology for future investments, the share price includes the market estimate 
of how much the firm will benefit from the technology ever after. Higher $r$ indicates the firm is able to generate more future cashflows for given a positive $\theta$. Thus, the market response coefficient $b_{\lambda}^{m}$ is strictly increasing in $r$. With higher market response coefficient, the firm has incentive to inflate the perceived profitability. This can be achieved by inflating initial investment, which increases the mean of either the input-based measure or the output-based measure. Therefore, the investment decision $I_{1}^{m}$ is strictly increasing in the parameter $r$.

Since the accounting measurements may not be perfectly precise, the market also responds to the measurement noise. As the variance of measurement noise $\sigma_{m}^{2}$ gets higher, the accounting reports are less informative, leading to a less responsive market price to the accounting report and the firm has less incentive to over-invest. Therefore, the initial investment $\left(I_{1}^{m}\right)$ is strictly decreasing in the measurement noise $\sigma_{m}^{2}$. Because the two exogenous parameters $\left(r\right.$ and $\left.\sigma_{m}^{2}\right)$ provide opposite incentives of investment choices, there exists a knife-edge case that the two opposite effects exactly offset each other, leading to the first-best initial investment.

\subsection{Comparing Output-based and Input-based Accounting Measures}

The following Corollary summarizes the properties of the equilibrium.

Corollary 3 If $\lambda^{O P}=2 \sqrt{\theta I_{1}}+\varepsilon^{O P}$ and $\lambda^{I P}=I_{1}+\varepsilon^{I P}$,

(i) if $\sigma_{O P}^{2}=2 r \sigma_{\theta}^{2}$, investment choice is the first-best level, and

(ii) if $\sigma_{I P}^{2}=(r+1) \sigma_{\theta}^{2}$, investment choice is the first-best level.

(iii) If $r>1(r<1)$, there exists a $\Sigma^{*}$ which lies in the interval between $(r+1) \sigma_{\theta}^{2}$ and $2 r \sigma_{\theta}^{2}$, such that a sufficient condition for input-based accounting to be more efficient than output-based accounting is $\sigma_{I P}^{2}=\sigma_{O P}^{2} \in\left[(r+1) \sigma_{\theta}^{2}, \Sigma^{*}\right]\left(\sigma_{I P}^{2}=\sigma_{O P}^{2} \in\left[\Sigma^{*},(r+1) \sigma_{\theta}^{2}\right]\right){ }^{18}$

The presence of the reinvestment option changes the market pricing and (thus) initial investment decisions. Compared with corresponding results in section 4 (see Theorem 4), the results are different in two ways. First, even with the output-based measure, investment efficiency is no longer monotonic in measurement noise. In particular, the output-based accounting measure does not perform best when the measure is noiseless. Second, the parameter $r$, a growth potential index so to speak, is important in determining economic efficiency, in addition to accounting rules and measurement errors. Intuitively, as $r$ increases, the market imposes more pressure on the accounting reports, and the firm is motivated to inflate the reports. ${ }^{19}$

\footnotetext{
${ }^{18}$ When $r=1$, we can show that output-based accounting is (weakly) more efficient than input-based accounting once $\beta$ is small enough.

${ }^{19}$ When $r=0$, the model reverts back to the basic model with output-based model performs best when noiseless.
} 
To see the trade-off precisely, we briefly review the mispricing structure. Substituting the pricing function $\left(P=a_{\lambda}^{O P}+b_{\lambda}^{O P} \lambda^{O P}\right)$ into the mispricing expression, we have

$$
\begin{aligned}
& E_{\varepsilon O P}\left[P-\left(2 \sqrt{\theta I_{1}}+r \theta\right) \mid \theta\right] \\
& =E_{\varepsilon} O P\left[a_{\lambda}^{O P}+b_{\lambda}^{O P}\left(2 \sqrt{\theta I_{1}}+\varepsilon^{O P}\right)-\left(2 \sqrt{\theta I_{1}}+r \theta\right) \mid \theta\right] \\
& =\left[b_{\lambda}^{O P}-1\right] 2 \sqrt{\theta I_{1}}-r \theta+a_{\lambda}^{O P}+E_{\varepsilon} O P\left[b_{\lambda}^{O P} \varepsilon^{O P} \mid \theta\right]
\end{aligned}
$$

The investment choice is first-best when the mispricing does not depend on the investment choice. Under the output-based measure, the mispricing is not a function of the initial investment only if the market response to accounting measure is equal to unity (i.e., $b_{\lambda}^{O P}=1$ ). However, in this modified model, a noiseless output-based measure will no longer lead to a unity market response. This is because, in this modified model, the market is pricing two streams of cashflows. First, for cashflows due to the initial investment, a unity response is needed with a noiseless measure. Second, for cashflows due to future investments, a non-zero response is needed. Combined the total response would be greater than unity in the noiseless case, thus providing ex ante incentive to deviate from the first-best investment.

Figure 3 provides an illustration of results in Corollary 3. Compared to Figure 2, the main difference is that the efficiency under the output-based measure peaks when the variance of the measure is not zero. Furthermore, when $r>1$, the peak occurs to the right of the peak under the input-based measure. This is because, relative to the input-based measure regime, a higher $r$ imposes more market pressure on the accounting measure and thus leading a more "distorted" investment choice.

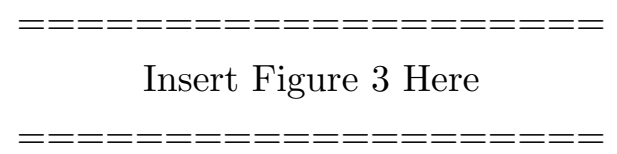

Finally, the extension leads us to rethinking the subtleties of output-based accounting when (re-investment) option value is important. From an accounting measurement perspective, $\lambda^{O P}$ can be viewed as a measure of "value in use," ignoring the option value of future use (through future investments). These measures do exist in accounting practices such as the re-valuation exercise in accounting for asset impairment. However, one may argue the option value would be impounded in a would-be exchange price of the asset in question. Fair value accounting measures, as proposed by the recent FASB exposure draft, may be close to having this characteristic. One can even argue these measures already exist in accounting practices such as the use of market value in initial and re-valuation of certain assets and in the initial recording of goodwill (provided the market prices are reflective of various option values). 


\section{Conclusion}

In this paper, we explore the trade-offs between two dominant accounting measurement bases: input-based measures and output-based measures. We discover that the trade-offs go beyond relevance and reliability issues commonly mentioned in accounting debates. We show that these two measures affect investment incentives in fundamentally different ways. The output-based measures have a natural advantage in aligning the firm and social investment incentives through a dampening effect, which limits over- and under-investment tendencies. However, high levels of noise and accounting manipulation, which are typically associated with output-based measures, may make output-based accounting far from a perfect solution to all accounting problems.

With an input-based accounting measurement basis, accounting numbers are less comprehensive, but their advantages are a lower level of noise and fewer accounting manipulation opportunities. In fact, being less comprehensive makes small but positive noise and/or manipulation desirable. Based on our analysis, the move toward output-based accounting, such as a fair value principle, may not be beneficial and requires more care and more extensive debates.

Our model is simple. Future works may benefit from including operating and financial choices and from analyzing more general settings with heterogeneous firms where accounting standards are central to an economic analysis of accounting. 


\section{Appendix}

Proof of Limit Properties of the Approximation Assumption. In this part of the appendix, we show that the approximation error, denoted $A E$, gets smaller and approaches zero as the mean of $z$ increases, for every realized value of the conditioning variable, $y+a z$. To prove this this formally, notice for every $W \equiv y+a z$,

$$
\begin{aligned}
A E(W) & =\int_{z>0} \int_{x}(x+z) f(x, z \mid y+a z) d x d z+G(0 \mid y+a z) \int_{x} x f(x \mid y) d x \\
& -\int_{z} \int_{x}(x+z) f(x, z \mid y+a z) d x d z \\
& =G(0 \mid y+a z) \int_{x} x f(x \mid y) d x-\int_{z<0} \int_{x}(x+z) f(x, z \mid y+a z) d x d z
\end{aligned}
$$

and we need to prove both components of the $A E(W)$ expression approach zero as $E[z]$ increases to every realization of $W \equiv y+a z$.

By assumption, the jointly distribution of $x$ and $y$ is

$$
\left[\begin{array}{l}
x \\
y
\end{array}\right] \sim N\left(\left[\begin{array}{l}
\mu_{x} \\
\mu_{y}
\end{array}\right],\left[\begin{array}{cc}
\sigma_{x}^{2} & \sigma_{x y} \\
\sigma_{x y} & \sigma_{y}^{2}
\end{array}\right]\right)
$$

and $z \backsim N\left[\mu_{z}, \sigma_{z}^{2}\right]$ is independent of $x$ and $y$. Hence, the jointly distribution of $x, z$ and $y+a z$ is

$$
\left[\begin{array}{c}
x \\
z \\
y+a z
\end{array}\right] \sim N\left(\left[\begin{array}{c}
\mu_{x} \\
\mu_{z} \\
\mu_{y}+a \mu_{z}
\end{array}\right],\left[\begin{array}{ccc}
\sigma_{x}^{2} & 0 & \sigma_{x y} \\
0 & \sigma_{z}^{2} & a \sigma_{z}^{2} \\
\sigma_{x y} & a \sigma_{z}^{2} & \sigma_{y}^{2}+a^{2} \sigma_{z}^{2}
\end{array}\right]\right)
$$

By the property of normal density function, the conditional distribution of $z$ given any realization of $W \equiv y+a z$ is

$$
z \mid W \sim N\left[\mu_{z}+\frac{a \sigma_{z}^{2}}{\sigma_{y}^{2}+a^{2} \sigma_{z}^{2}}\left(W-\mu_{y}-a \mu_{z}\right), \frac{\sigma_{z}^{2} \sigma_{y}^{2}}{\sigma_{y}^{2}+a^{2} \sigma_{z}^{2}}\right]
$$

To simplify the notation, we denote the above by $z \mid W \backsim N\left[\mu_{z^{\prime}}, \sigma_{z^{\prime}}^{2}\right]$. And the jointly conditional distribution of $x$ and $z$ given any realization of $W$ is

$$
\left.\left[\begin{array}{l}
x \\
z
\end{array}\right] \text { W }\right] \sim N\left(\left[\begin{array}{c}
\mu_{x}+\frac{\sigma_{x y}}{\sigma_{y}^{2}+a^{2} \sigma_{z}^{2}}\left(W-\mu_{y}-a \mu_{z}\right) \\
\mu_{z}+\frac{a \sigma_{z}^{2}}{\sigma_{y}^{2}+a^{2} \sigma_{z}^{2}}\left(W-\mu_{y}-a \mu_{z}\right)
\end{array}\right],\left[\begin{array}{cc}
\sigma_{x}^{2}-\frac{\sigma_{x y}^{2}}{\sigma_{y}^{2}+a^{2} \sigma_{z}^{2}} & -\frac{\sigma_{x y} a \sigma_{z}^{2}}{\sigma_{y}^{2}+a^{2} \sigma_{z}^{2}} \\
-\frac{\sigma_{x y} a \sigma_{z}^{2}}{\sigma_{y}^{2}+a^{2} \sigma_{z}^{2}} & \frac{\sigma_{z}^{2} \sigma_{y}^{2}}{\sigma_{y}^{2}+a^{2} \sigma_{z}^{2}}
\end{array}\right]\right)
$$

Again to simplify, we denote the above by $\left[\begin{array}{c}x \\ z\end{array} \mid W\right] \sim N\left(\left[\begin{array}{c}\mu_{x^{\prime}} \\ \mu_{z^{\prime}}\end{array}\right],\left[\begin{array}{cc}\sigma_{x^{\prime}}^{2} & \sigma_{x z} \\ \sigma_{x z} & \sigma_{z^{\prime}}^{2}\end{array}\right]\right)$ 
Using (32), the conditional cumulative density function of $z, G(z \mid W)=\Phi\left(\frac{z-\mu_{z^{\prime}}}{\sigma_{z^{\prime}}}\right)$, where $\Phi(\cdot)$ is the standard normal cumulative distribution function (cdf). Hence, $G(0 \mid W)=\Phi\left(\frac{-\mu_{z^{\prime}}}{\sigma_{z^{\prime}}}\right)$. If $\mu_{z}$ increases, $\frac{-\mu_{z^{\prime}}}{\sigma_{z^{\prime}}}$ goes to negative infinity because, $\mu_{z^{\prime}} \equiv \frac{\sigma_{y}^{2}}{\sigma_{y}^{2}+a^{2} \sigma_{z}^{2}} \mu_{z}+\frac{a \sigma_{z}^{2}}{\sigma_{y}^{2}+a^{2} \sigma_{z}^{2}}\left(W-\mu_{y}\right)$, for any given $W$. By the property of standard normal cumulative density function $(\Phi(\cdot)), \Phi\left(\frac{-\mu_{z^{\prime}}}{\sigma_{z^{\prime}}}\right) \rightarrow 0$ as $\frac{-\mu_{z^{\prime}}}{\sigma_{z^{\prime}}} \rightarrow-\infty$. Therefore, the first component of $(31), G(0 \mid W) \int_{x} x f(x \mid y) d x$, goes to zero.

Using (33), the second component of (31) is

$$
\begin{aligned}
& \int_{z<0} \int_{x}(x+z) f(x, z \mid W \equiv y+a z) d x d z \\
& =\int_{z<0} \int_{x}(x+z) f(z \mid W) f(x \mid z, W) d x d z \\
& =\int_{z<0}\left[\int_{x} x f(x \mid z, W) d x+z \int_{x} f(x \mid z, W) d x\right] f(z \mid W) d z \\
& =\int_{z<0}\left[\mu_{x^{\prime}}+\frac{\sigma_{x z}}{\sigma_{z^{\prime}}^{2}}\left(z-\mu_{z^{\prime}}\right)+z\right] f(z \mid W) d z \\
& =\left[\mu_{x}+\frac{\sigma_{x y}}{\sigma_{y}^{2}}\left(W-\mu_{y}\right)\right] G(0 \mid W)+\left(1-\frac{a \sigma_{x y}}{\sigma_{y}^{2}}\right) \int_{z<0} z f(z \mid W) d z
\end{aligned}
$$

Similar to the first component, the first part of second component, $\left[\mu_{x}+\frac{\sigma_{x y}}{\sigma_{y}^{2}}\left(W-\mu_{y}\right)\right] G(0 \mid W)$, also approaches zero when $\mu_{z}$ increases.

Finally, we need to find show the second part of the second component, $\int_{z<0} z f(z \mid W) d z$, goes to zero as $\mu_{z}$ increases.

$$
\begin{aligned}
\int_{z<0} z f(z \mid W) d z & =\frac{1}{\sigma_{z^{\prime}} \sqrt{2 \pi}} \int_{-\infty}^{0} z \exp \left(-\frac{\left(z-\mu_{z^{\prime}}\right)^{2}}{2 \sigma_{z^{\prime}}^{2}}\right) d z \\
& =\frac{1}{\sigma_{z^{\prime}} \sqrt{2 \pi}} \int_{-\infty}^{0} z \exp \left(-\frac{z^{2}}{2 \sigma_{z^{\prime}}^{2}}\right) \cdot \exp \left(\frac{z \mu_{z^{\prime}}}{\sigma_{z^{\prime}}^{2}}\right) \cdot \exp \left(-\frac{\mu_{z^{\prime}}^{2}}{2 \sigma_{z^{\prime}}^{2}}\right) d z
\end{aligned}
$$

Because $z$ is always negative, $\exp \left(\frac{z \mu_{z^{\prime}}}{\sigma_{z^{\prime}}^{2}}\right)$ must be positive and always smaller than one. So the absolute value of above expression must be smaller than the absolute value of $\frac{1}{\sigma_{z^{\prime}} \sqrt{2 \pi}} \int_{-\infty}^{0} z \exp \left(-\frac{z^{2}}{2 \sigma_{z^{\prime}}^{2}}\right)$. $\exp \left(-\frac{\mu_{z^{\prime}}^{2}}{2 \sigma_{z^{\prime}}^{2}}\right) d z=\frac{\sigma_{z^{\prime}}}{\sqrt{2 \pi}} \exp \left(-\frac{\mu_{z^{\prime}}^{2}}{2 \sigma_{z^{\prime}}^{2}}\right)$. As the mean of $\mu_{z}$ increases, $-\frac{\mu_{z^{\prime}}^{2}}{2 \sigma_{z^{\prime}}^{2}}$ goes to negative infinity. Hence the absolute value of $\frac{1}{\sigma_{z^{\prime}} \sqrt{2 \pi}} \int_{-\infty}^{0} z \exp \left(-\frac{z^{2}}{2 \sigma_{z^{\prime}}^{2}}\right) \cdot \exp \left(-\frac{\mu_{z^{\prime}}^{2}}{2 \sigma_{z^{\prime}}^{2}}\right) d z$ goes to zero, and thus, the second part of the second component, $\int_{z<0} z f(z \mid W) d z$, also approaches zero.

To summarize, we have shown for every $W \equiv y+a z$, as $\mu_{z}$ increases, $A E(W)$ approaches zero. 
Proof of Theorem 1. We begin with the linear pricing conjecture:

$$
P\left(z_{1}\right)=a+b z_{1}
$$

The manager's maximization program becomes:

$$
\begin{aligned}
& \text { Choose } I(\theta) \text { to } \max \int_{\theta} V(\theta \mid I(\cdot)) G(\theta) d \theta \\
& =\int_{\theta} E_{x_{1} x_{2}}\left[-I+\beta P+(1-\beta)\left(z_{1}+z_{2}\right)\right] G(\theta) d \theta \\
& =-I+\beta\left(a+b\left(\mu+\int_{\theta} k \sqrt{\theta I} G(\theta) d \theta\right)\right)+(1-\beta)\left(2 \mu+\int_{\theta} 2 \sqrt{\theta I} G(\theta) d \theta\right)
\end{aligned}
$$

The point-wise first-order condition with respect to $I$ is, for $\theta>0$,

$$
\begin{aligned}
0 & =-1+\frac{\left(1-\beta+\frac{\beta b k}{2}\right) \sqrt{\theta}}{\sqrt{I}} \\
I & =\left(1-\beta+\frac{\beta b k}{2}\right)^{2} \theta \\
& =\left(1+\beta\left(\frac{b k}{2}-1\right)\right)^{2} \theta
\end{aligned}
$$

and for $\theta<0, I=0$. So, it must be the case that

$$
\delta=\left(1+\beta\left(\frac{b k}{2}-1\right)\right)^{2}
$$

Given that $\delta$ and $k$ are constants,

$$
\left(\begin{array}{c}
x_{1}+x_{2}+2 \sqrt{\delta} \theta \\
x_{1}+k \sqrt{\delta} \theta
\end{array}\right) \sim N\left(\left(\begin{array}{c}
2 \mu+2 \sqrt{\delta} \theta_{0} \\
\mu+k \sqrt{\delta} \theta_{0}
\end{array}\right),\left[\begin{array}{cc}
2(1+\alpha) \sigma^{2}+4 \delta \sigma_{\theta}^{2} & (1+\alpha) \sigma^{2}+2 k \delta \sigma_{\theta}^{2} \\
(1+\alpha) \sigma^{2}+2 k \delta \sigma_{\theta}^{2} & \sigma^{2}+k^{2} \delta \sigma_{\theta}^{2}
\end{array}\right]\right)
$$


So, we have the approximate pricing function, using (6) :

$$
\begin{aligned}
P & =E\left[z_{1}+z_{2} \mid z_{1}\right]=E\left[x_{1}+x_{2}+2 \sqrt{\theta I} \mid x_{1}+k \sqrt{\theta I}\right] \\
& \tilde{=} E\left[x_{1}+x_{2}+2 \theta \sqrt{\delta} \mid x_{1}+k \theta \sqrt{\delta}\right] \\
& =2 \mu+2 \sqrt{\delta} \theta_{0}+\frac{(1+\alpha) \sigma^{2}+2 k \delta \sigma_{\theta}^{2}}{\sigma^{2}+k^{2} \delta \sigma_{\theta}^{2}}\left(z_{1}-\mu-k \sqrt{\delta} \theta_{0}\right) \\
& =\frac{(1-\alpha) \sigma^{2}+2\left(k^{2}-k\right) \delta \sigma_{\theta}^{2}}{\sigma^{2}+k^{2} \delta \sigma_{\theta}^{2}} \mu+\frac{(2-(1+\alpha) k) \sigma^{2}}{\sigma^{2}+k^{2} \delta \sigma_{\theta}^{2}} \sqrt{\delta} \theta_{0} \\
& +\frac{(1+\alpha) \sigma^{2}+2 k \delta \sigma_{\theta}^{2}}{\sigma^{2}+k^{2} \delta \sigma_{\theta}^{2}} z_{1}
\end{aligned}
$$

So, it must be the case that

$$
\begin{aligned}
& a=(2-b) \mu+(2-k b) \sqrt{\delta} \theta_{0} \\
& b=\frac{(1+\alpha) \sigma^{2}+2 k \delta \sigma_{\theta}^{2}}{\sigma^{2}+k^{2} \delta \sigma_{\theta}^{2}}
\end{aligned}
$$

To show the existence of $\delta$ and $b$, substituting $b$ into $\delta$, and simplifying, we have

$$
(\sqrt{\delta}-1) \cdot\left(k^{2} \delta \frac{\sigma_{\theta}^{2}}{\sigma^{2}}+1\right)=\beta\left[\frac{k(1+\alpha)}{2}-1\right]
$$

As $\beta \in[0,1], k \in[0,2]$, and $\alpha \in(-1,1)$, the range of the right-hand-side is from -1 to 1 . As $\delta>0$, and all of the functions are continuous, the left-hand-side covers the range from -1 to $+\infty$. Therefore, there is at least one positive root of $\delta .{ }^{20}$

Proof of Theorem 2. Suppose the manager chooses the first-best investment $\delta=1$. Correspondingly, using (7), (8), and (9) the market pricing function would be

$$
\begin{aligned}
P\left(z_{1}\right) & =a+b_{\delta=1} \times z_{1}, \text { where } \\
b_{\delta=1} & =\frac{(1+\alpha) \sigma^{2}+2 k \sigma_{\theta}^{2}}{\sigma^{2}+k^{2} \sigma_{\theta}^{2}}, a=\left(2-b_{\delta=1}\right) \mu+\left(2-k b_{\delta=1}\right) \theta_{0}
\end{aligned}
$$

To sustain the equilibrium, the manager's reaction to $b_{\delta=1}=\frac{(1+\alpha) \sigma^{2}+2 k \sigma_{\theta}^{2}}{\sigma^{2}+k^{2} \sigma_{\theta}^{2}}$ must indeed be to set $\delta=1$, which is the first-best investment level. That is, it must be the case that

$$
\left.\delta\right|_{b_{\delta=1}, k=k^{*}}=1
$$

\footnotetext{
${ }^{20}$ Technically, in some rare cases (when the right-hand-side is very close to -1 ), the function could have three positive roots of $\delta$. Then, in our study, we only consider the root that is closest to one. That is, in multiple equilibrium cases, the economy chooses the most efficient one.
} 
Using (11), we have

$$
\begin{aligned}
\left.\delta\right|_{b_{\delta=1}, k=k^{*}} & =\left(1+\beta\left(\frac{b_{\delta=1} k^{*}}{2}-1\right)\right)^{2} \\
& =\left(1+\beta\left(\frac{(1+\alpha) \sigma^{2}+2 \frac{2}{1+\alpha} \sigma_{\theta}^{2}}{\sigma^{2}+\left(\frac{2}{1+\alpha}\right)^{2} \sigma_{\theta}^{2}} \cdot \frac{2}{1+\alpha}-1\right)\right)^{2} \\
& =1
\end{aligned}
$$

Generally, we re-write $b k$ as the following

$$
\begin{aligned}
b k & =\frac{(1+\alpha) \sigma^{2}+2 k \delta \sigma_{\theta}^{2}}{\sigma^{2}+k^{2} \delta \sigma_{\theta}^{2}} \cdot k \\
& =2+\frac{[(1+\alpha) k-2] \sigma^{2}}{\sigma^{2}+k^{2} \delta \sigma_{\theta}^{2}}
\end{aligned}
$$

So

$$
\begin{aligned}
\delta & =\left(1+\beta\left(\frac{b k}{2}-1\right)\right)^{2} \\
& =\left(1+\frac{\beta}{2} \cdot \frac{[(1+\alpha) k-2] \sigma^{2}}{\sigma^{2}+k^{2} \delta \sigma_{\theta}^{2}}\right)^{2}
\end{aligned}
$$

Now it is clear that if $k>k^{*}, \delta>1$, and $k<k^{*}, \delta<1$.

Proposition 1 Comparative Statics in the Basic Setup

1. $\delta$ and $b$ strictly increase in $\alpha$;

2. When $k>k^{*}\left(k<k^{*}\right)$, $\delta$ and $b$ strictly decrease in $\frac{\sigma_{\theta}^{2}}{\sigma^{2}}$ (strictly increase in $\left.\frac{\sigma_{\theta}^{2}}{\sigma^{2}}\right)$; and

3. When $k>k^{*}\left(k<k^{*}\right)$, $\delta$ strictly increases in $\beta$ (strictly decreases in $\beta$ ). Also $b$ strictly decreases in $\beta$ for any $k \neq k^{*}$.

Proof of Proposition 1. Using the pricing function (8), substituting $b$ into the manager's investment decision (11), we have the following equation which has to hold in equilibrium.

$$
(\sqrt{\delta}-1) \cdot\left(k^{2} \delta \frac{\sigma_{\theta}^{2}}{\sigma^{2}}+1\right)=\beta\left[\frac{k(1+\alpha)}{2}-1\right]
$$


The left-hand-side increases monotonically in $\delta^{21}$, and is independent of $\alpha$. The right-hand-side increases monotonously in $\alpha$, and is independent of $\delta$. It is easy to see that $\delta$ strictly increases in $\alpha$. By (11), $b$ strictly increases in $\delta$, therefore, $b$ strictly increases in $\alpha$. From Theorem $2, \delta>1$ when $k>k^{*}$. Given the equation above, the right-hand-side is positive as $k>k^{*}$. As $\frac{\sigma_{\theta}^{2}}{\sigma^{2}}$ increases, $\delta$ has to decrease to let the equation sustain. Similarly if $k<k^{*}, \delta$ increases in $\frac{\sigma_{\theta}^{2}}{\sigma^{2}}$. By (11), $b$ changes in the same way as $\delta$. As we show above, the left-hand-side increases monotonously in $\delta$. When $k>k^{*}$, the right-hand-side increases in $\beta$, so $\delta$ strictly increases in $\beta$. Similarly, $\delta$ strictly decreases in $\beta$, when $k<k^{*}$, and $\delta$ is equal to one regardless of $\beta$, when $k=k^{*}$. To analyze the change of $b$, re-write (8) as the following,

$$
b=\frac{2}{k}+\frac{(1+\alpha)-\frac{2}{k}}{1+k^{2} \delta \frac{\sigma_{\theta}^{2}}{\sigma^{2}}}
$$

If $k>k^{*},(1+\alpha)-\frac{2}{k}>0$. As $\beta$ increases, $\delta$ increases from the above analysis. Therefore, $b$ decreases in $\beta$. Similarly, $b$ also strictly decreases in $\beta$, when $k<k^{*}$,and is equal to $1+\alpha$ regardless of $\beta$, when $k=k^{*}$.

Remarks: The first result is quite straight-forward. As $\alpha$ increases, the short-term and longterm cash flows from the on-going activities are more correlated. Therefore, the market response coefficient $b$ increases, and the firm has an incentive to invest more to inflate the market price. That is, $\delta$ increases.

Combined with Theorem 2, the second comparative static result shows that a higher $\frac{\sigma_{\theta}^{2}}{\sigma^{2}}$ can induce a more efficient investment level (i.e., pushing $\delta$ closer to one). As $\sigma^{2}$ gets lower, the on-going cash flows are less noisy, making the aggregate cash flow report $\left(z_{1}=x_{1}+k \sqrt{\theta I}\right)$ more informative about the short-term investment return $(k \sqrt{\theta I})$. Intuitively, it is easier for the market to identify the first-period investment return. (Technically, the market response coefficient is closer to $\frac{2}{k}$ as $\frac{\sigma_{\theta}^{2}}{\sigma^{2}}$ increases.) As a result, the short-term investment return is less mispriced while the on-going cash flow is more mispriced. However, given that the mispricing of the on-going cash flow does not have any negative effect on the real investment decision, an increase in $\frac{\sigma_{\theta}^{2}}{\sigma^{2}}$ improves the investment efficiency. In the extreme, when $\sigma^{2}$ approaches zero, the equilibrium achieves first-best results.

With short-term pressure $(\beta>0)$, the firm investment deviates from the first-best (by Theorem 2 ). The magnitude of the deviation increases in market pressure $(\beta)$. As the firm makes less efficient investment under more market pressure, the market responds less to the cash flow report ( $b$ decreases in $\beta$ ) because the value lost due to inefficient investment increases in $\beta$.

Proof of Theorem 3. Suppose the firm chooses an input-based accounting system, based on the linear conjecture:

\footnotetext{
${ }^{21}$ The monotonicity is based on the previous assumption that in the case of multiple roots for the equilibrium $\delta$, the economy chooses the root with which the investment is most efficient. See proof of Theorem 1.
} 


$$
P\left(z_{1}, y^{I P}\right)=a^{I P}+b_{z}^{I P} \times z_{1}+b_{y}^{I P} \times y^{I P}
$$

The manager maximizes the expected payoff:

$$
\begin{aligned}
& \text { Choose } I(\theta) \text { to } \max \int_{\theta} V(\theta \mid I(\cdot)) G(\theta) d \theta \\
& =\int_{\theta} E_{x_{1} x_{2}}\left[-I+\beta P+(1-\beta)\left(z_{1}+z_{2}\right)\right] G(\theta) d \theta \\
& =-I+\beta\left(a^{I P}+b_{z}^{I P}\left(\mu+\int_{\theta} k \sqrt{\theta I} G(\theta) d \theta\right)+b_{y}^{I P} I\right)+(1-\beta)\left(2 \mu+\int_{\theta} 2 \sqrt{\theta I} G(\theta) d \theta\right)
\end{aligned}
$$

The point-wise first-order condition with respect to $I$ is for $\theta>0$, and we have

$$
\begin{aligned}
0 & =-1+\frac{\left(1-\beta+\frac{\beta k b_{z}^{I P}}{2}\right) \sqrt{\theta}}{\sqrt{I}}+\beta b_{y}^{I P} \\
I^{I P} & =\left(\frac{1-\beta+\frac{\beta k b_{z}^{I P}}{2}}{1-\beta b_{y}^{I P}}\right)^{2} \theta
\end{aligned}
$$

and for $\theta<0, I^{I P}=0$.

So it must be the case that

$$
\delta_{I P}=\left(\frac{1-\beta+\frac{\beta k b_{z}^{I P}}{2}}{1-\beta b_{y}^{I P}}\right)^{2}
$$

Using (21), (22), we have $\left(\begin{array}{c}x_{1}+x_{2}+2 \sqrt{\delta_{I P}} \theta \\ x_{1}+k \sqrt{\delta_{I P}} \theta \\ \varepsilon^{I P}+\delta_{I P} \theta\end{array}\right)$ is normally distributed with mean

$$
\left(\begin{array}{c}
2 \mu+2 \sqrt{\delta_{I P}} \theta_{0} \\
\mu+k \sqrt{\delta_{I P}} \theta_{0} \\
\delta_{I P} \theta_{0}
\end{array}\right)
$$

and variance

$$
\left[\begin{array}{ccc}
2(1+\alpha) \sigma^{2}+4 \delta_{I P} \sigma_{\theta}^{2} & (1+\alpha) \sigma^{2}+2 k \delta_{I P} \sigma_{\theta}^{2} & 2 \delta_{I P}^{\frac{3}{2}} \sigma_{\theta}^{2} \\
(1+\alpha) \sigma^{2}+2 k \delta_{I P} \sigma_{\theta}^{2} & \sigma^{2}+k^{2} \delta_{I P} \sigma_{\theta}^{2} & k \delta_{I P}^{2} \sigma_{\theta}^{2} \\
2 \delta_{I P}^{\frac{3}{2}} \sigma_{\theta}^{2} & k \delta_{I P}^{\frac{3}{2}} \sigma_{\theta}^{2} & \delta_{I P}^{2} \sigma_{\theta}^{2}+\sigma_{I P}^{2}
\end{array}\right]
$$


Using the approximation assumption (6), the pricing function becomes

$$
\begin{aligned}
& P=E\left[z_{1}+z_{2} \mid z_{1}, y^{I P}\right]=E\left[x_{1}+x_{2}+2 \sqrt{\theta I} \mid x_{1}+k \sqrt{\theta I}, I+\varepsilon^{I P}\right] \\
& \tilde{=} E\left[x_{1}+x_{2}+2 \theta \sqrt{\delta} \mid x_{1}+k \theta \sqrt{\delta}, \delta \theta+\varepsilon^{I P}\right] \\
& =2 \mu+2 \sqrt{\delta_{I P}} \theta_{0}+\left[(1+\alpha) \sigma^{2}+2 k \delta_{I P} \sigma_{\theta}^{2}, 2 \delta_{I P}^{\frac{3}{2}} \sigma_{\theta}^{2}\right] \text {. } \\
& {\left[\begin{array}{cc}
\sigma^{2}+k^{2} \delta_{I P} \sigma_{\theta}^{2} & k \delta_{I P}^{\frac{3}{2}} \sigma_{\theta}^{2} \\
k \delta_{I P}^{\frac{3}{2}} \sigma_{\theta}^{2} & \delta_{I P}^{2} \sigma_{\theta}^{2}+\sigma_{I P}^{2}
\end{array}\right]^{-1} \cdot\left[\begin{array}{c}
z_{1}-\mu-k \sqrt{\delta_{I P}} \theta_{0} \\
y^{I P}-\delta_{I P} \theta_{0}
\end{array}\right]} \\
& =2 \mu+2 \sqrt{\delta_{I P}} \theta_{0}+\frac{(1+\alpha) \delta_{I P}^{2} \sigma^{2} \sigma_{\theta}^{2}+(1+\alpha) \sigma^{2} \sigma_{I P}^{2}+2 k \delta_{I P} \sigma_{I P}^{2} \sigma_{\theta}^{2}}{\delta_{I P}^{2} \sigma^{2} \sigma_{\theta}^{2}+\sigma^{2} \sigma_{I P}^{2}+k^{2} \delta_{I P} \sigma_{I P}^{2} \sigma_{\theta}^{2}} \cdot\left(z_{1}-\mu-k \sqrt{\delta_{I P}} \theta_{0}\right) \\
& +\frac{[2-(1+\alpha) k] \delta_{I P}^{\frac{3}{2}} \sigma^{2} \sigma_{\theta}^{2}}{\delta_{I P}^{2} \sigma^{2} \sigma_{\theta}^{2}+\sigma^{2} \sigma_{I P}^{2}+k^{2} \delta_{I P} \sigma_{I P}^{2} \sigma_{\theta}^{2}} \cdot\left(y^{I P}-\delta_{I P} \theta_{0}\right)
\end{aligned}
$$

So it must be the case that

$$
\begin{aligned}
& b_{z}^{I P}=\frac{(1+\alpha) \delta_{I P}^{2} \sigma^{2} \sigma_{\theta}^{2}+(1+\alpha) \sigma^{2} \sigma_{I P}^{2}+2 k \delta_{I P} \sigma_{I P}^{2} \sigma_{\theta}^{2}}{\delta_{I P}^{2} \sigma^{2} \sigma_{\theta}^{2}+\sigma^{2} \sigma_{I P}^{2}+k^{2} \delta_{I P} \sigma_{I P}^{2} \sigma_{\theta}^{2}} \\
& b_{y}^{I P}=\frac{[2-(1+\alpha) k] \delta_{I P}^{\frac{3}{2}} \sigma^{2} \sigma_{\theta}^{2}}{\delta_{I P}^{2} \sigma^{2} \sigma_{\theta}^{2}+\sigma^{2} \sigma_{I P}^{2}+k^{2} \delta_{I P} \sigma_{I P}^{2} \sigma_{\theta}^{2}} \\
& a^{I P}=\left(2-b_{z}^{I P}\right) \mu+\left(2-k b_{z}^{I P}-\sqrt{\delta_{I P}} b_{y}^{I P}\right) \sqrt{\delta_{I P}} \theta_{0}
\end{aligned}
$$

To show the existence of $\delta_{I P}, b_{z}^{I P}$ and $b_{y}^{I P}$, substituting $b_{z}^{I P}(19)$ and $b_{y}^{I P}(20)$ into the investment decision (22), we obtain

$$
\left(\sqrt{\delta_{I P}}-1\right) \cdot\left(k^{2} \delta_{I P} \frac{\sigma_{\theta}^{2}}{\sigma^{2}}+1+\frac{\delta_{I P}^{2} \sigma_{\theta}^{2}}{\sigma_{I P}^{2}}\right)=\beta\left[\frac{k(1+\alpha)}{2}-1\right] \cdot\left(1-\frac{\delta_{I P}^{2} \sigma_{\theta}^{2}}{\sigma_{I P}^{2}}\right)
$$

As $\delta_{I P} \in(0,+\infty)$, and the all functions are continuous, the left-hand-side at least covers the range from -1 to $+\infty$. The range of the left-hand-side could be larger depending on the parameter space. The right-hand-side (a parabola) at least covers the range $\left(\beta\left[\frac{k(1+\alpha)}{2}-1\right],-\infty\right)$. We showed earlier that the range of $\beta\left[\frac{k(1+\alpha)}{2}-1\right]$ is from -1 to 1 . So the left-hand-side curve and the right- handside curve must intersect at least once in $\delta_{I P} \in(0,+\infty)$. Therefore, there is at least one positive root of $\delta_{I P}$.

Using a similar method, we obtain the linear equilibrium under output-based accounting.

Proof of Corollary 1. From Theorem 3, using the pricing function (16) and, substituting $b_{z}^{O P}$ (17) and $b_{y}^{O P}(18)$ into the manager's investment decision (22), we obtain the following equation, 
which has to hold in equilibrium under the output-based accounting,

$$
\left(\sqrt{\delta_{O P}}-1\right) \cdot\left(k^{2} \delta_{O P} \frac{\sigma_{\theta}^{2}}{\sigma_{O P}^{2}}+1+k^{2} \delta_{O P} \frac{\sigma_{\theta}^{2}}{\sigma^{2}}\right)=\beta\left[\frac{k(1+\alpha)}{2}-1\right]
$$

The second parenthesis on the left-hand-side of the equation is always positive and decreases in $\sigma_{O P}^{2}$. So, the absolute value of $\left(\sqrt{\delta_{O P}}-1\right)$ must increase in $\sigma_{O P}^{2}$, (given that the right- hand-side is a constant,) which indicates better investment choices as $\sigma_{O P}^{2}$ is lower. As the sign of $\left(\sqrt{\delta_{O P}}-1\right)$ is the same as the right-hand-side, $\delta_{O P}$ increases (decreases) in $\sigma_{O P}^{2}$ when $k>k^{*}\left(k<k^{*}\right)$.

For input-based accounting, we substitute $b_{z}^{I P}(19)$ and $b_{y}^{I P}(20)$ into the manager's investment decision (22), and we obtain the following equation:

$$
\left(\sqrt{\delta_{I P}}-1\right) \cdot\left(k^{2} \delta_{I P} \frac{\sigma_{\theta}^{2}}{\sigma^{2}}+1+\frac{\delta_{I P}^{2} \sigma_{\theta}^{2}}{\sigma_{I P}^{2}}\right)=\beta\left[\frac{k(1+\alpha)}{2}-1\right] \cdot\left(1-\frac{\delta_{I P}^{2} \sigma_{\theta}^{2}}{\sigma_{I P}^{2}}\right)
$$

Suppose $k>k^{*}$. The right-hand-side must be positive when $\sigma_{I P}^{2}$ is sufficiently high, which means the left-hand-side is positive or $\delta_{I P}>1$ or the firm overinvests. As $\sigma_{I P}^{2}$ is reduced, the righthand-side decreases and the second parenthesis on the left-hand-side increases. Thus, to maintain equation $(35), \delta_{I P}$ has to be reduced, mitigating the investment inefficiency. Once $\sigma_{I P}^{2}$ is reduced to $\sigma_{\theta}^{2}$, the equilibrium reaches the first-best, $\delta_{I P}=1$. However, as $\sigma_{I P}^{2}$ continues to decrease past $\sigma_{\theta}^{2}$, the right-hand-side turns negative, and $\delta_{I P}$ has to be lower than one to sustain equation (35). The investment $\delta_{I P}$ becomes less as $\sigma_{I P}^{2}$ continues to decrease. We get similar results in the case of $k<k^{*}$. Thus, $\delta_{I P}$ increases (decreases) in $\sigma_{I P}^{2}$ when $k>k^{*}\left(k<k^{*}\right)$.

From Theorem 3, using (17), (18), (19), and (20), we can readily find the effect of $\sigma_{m}^{2}$ on the market response coefficients $b_{z}^{m}$ and $b_{y}^{m}$.

Proof of Theorem 4. To prove claim (i), substituting $\sigma_{O P}^{2}=0$ into (17) and (18), we have $b_{z}^{O P}=1+\alpha$, and $b_{y}^{O P}=\frac{2}{k}-(1+\alpha)$. Then using $(22)$, the investment level is first-best $\left(\delta_{O P}=1\right)$.

To prove claim (ii), suppose that the manager chooses the first-best investment level $I=\theta$ under input-based accounting. Correspondingly, using (16), (19), and (20), the market pricing function would be

$$
\begin{aligned}
P^{I P}\left(z_{1}, y^{I P}\right) & =a^{I P}+b_{z, \delta_{a}=1}^{I P} \times z_{1}+b_{y, \delta_{a}=1}^{I P} \times y^{I P}, \text { where } \\
b_{z, \delta_{a}=1}^{I P} & =\frac{(1+\alpha) \sigma^{2}\left(\sigma_{\theta}^{2}+\sigma_{I P}^{2}\right)+2 k \sigma_{I P}^{2} \sigma_{\theta}^{2}}{\sigma^{2}\left(\sigma_{\theta}^{2}+\sigma_{I P}^{2}\right)+k^{2} \sigma_{I P}^{2} \sigma_{\theta}^{2}} \\
b_{y, \delta_{a}=1}^{I P} & =\frac{[2-(1+\alpha) k] \sigma^{2} \sigma_{\theta}^{2}}{\sigma^{2}\left(\sigma_{\theta}^{2}+\sigma_{I P}^{2}\right)+k^{2} \sigma_{I P}^{2} \sigma_{\theta}^{2}}
\end{aligned}
$$

To sustain the equilibrium, the manager's reaction to $b_{z, \delta_{a}=1}^{I P}$ and $b_{y, \delta_{a}=1}^{I P}$ must, indeed, be to set 
the investment at the first-best level. That is, it must be the case that

$$
\left.\delta_{I P}\right|_{b_{z, \delta_{a}=1}^{I P}, b_{y, \delta_{a}=1}^{I P}}=1
$$

By assuming $\sigma_{I P}^{2}=\sigma_{\theta}^{2}$, we have

$$
\begin{aligned}
& b_{z, \delta_{a}=1}^{I P}=\frac{2(1+\alpha) \sigma^{2} \sigma_{\theta}^{2}+2 k \sigma_{\theta}^{4}}{2 \sigma^{2} \sigma_{\theta}^{2}+k^{2} \sigma_{\theta}^{4}}, \\
& b_{y, \delta_{a}=1}^{I P}=\frac{[2-(1+\alpha) k] \sigma^{2} \sigma_{\theta}^{2}}{2 \sigma^{2} \sigma_{\theta}^{2}+k^{2} \sigma_{\theta}^{4}}
\end{aligned}
$$

Using (22), we have

$$
\delta_{I P}=\left(\frac{1-\beta+\frac{\beta k b_{z}^{I P}}{2}}{1-\beta b_{y}^{I P}}\right)^{2}
$$

Substituting $b_{z, \delta_{a}=1}^{I P}$ and $b_{y, \delta_{a}=1}^{I P}$ into the above expression yields:

$$
\begin{aligned}
\left.\delta_{I P}\right|_{b_{z, \delta_{a}=1}^{I P}, b_{y, \delta_{a}=1}^{I P}} & =\left(\frac{1+\beta \frac{[(1+\alpha) k-2] \sigma^{2} \sigma_{\theta}^{2}}{2 \sigma^{2} \sigma_{\theta}^{2}+k^{2} \sigma_{\theta}^{4}}}{1-\beta \frac{[2-(1+\alpha) k] \sigma^{2} \sigma_{\theta}^{2}}{2 \sigma^{2} \sigma_{\theta}^{2}+k^{2} \sigma_{\theta}^{4}}}\right)^{2} \\
& =1
\end{aligned}
$$

Claim (iii) and (iv) compare the investment efficiency under different accounting basis. Recall the expected net return of the project is, using (21)

$$
E\left[R^{m}\right]=E\left[f_{1}\left(\theta, I^{m}\right)+f_{2}\left(\theta, I^{m}\right)-I^{m}\right]=E\left[2 \sqrt{\theta I^{m}}-I^{m}\right]=\left[1-\left(\sqrt{\delta_{m}}-1\right)^{2}\right] \cdot \theta_{0}
$$

It is clear from (36) that $E\left[R^{m}\right]$ is single-peaked at $\delta_{m}=1, m \in\{I P, O P\}$.

To prove claim (iii), invoke claim (ii) of Theorem 4: $\delta_{I P}=1$ when $\sigma_{I P}^{2}=\sigma_{\theta}^{2}$; and invoke claim (ii) of Corollary (1): $\delta_{I P}$ is monotonic in $\sigma_{I P}^{2}$ (increasing or decreasing depending on $k$ ). Combined, we must have that $E\left[R^{I P}\right]$ is single-peaked at $\sigma_{I P}^{2}=\sigma_{\theta}^{2}$ regardless of $k$.

Next, we compare $E\left[R^{I P}\right]$ in the two extreme cases: (1) the case without the input-based accounting measures (or the case $\sigma_{I P}^{2}=+\infty$ by Corollary 1 claim (i)), and (2) the case of a perfectly precise input-based accounting measures $\left(\sigma_{I P}^{2}=0\right)$. Substituting $\sigma_{I P}^{2}=+\infty$ into (19), and (20), we have $b_{z}^{I P}=\frac{(1+\alpha) \sigma^{2}+2 k \delta_{I P} \sigma_{\theta}^{2}}{\sigma^{2}+k^{2} \delta_{I P} \sigma_{\theta}^{2}}$, and $b_{y}^{I P}=0$. Then using (22), we have,

$$
\sqrt{\delta_{I P}}=1+\frac{\beta\left[\frac{k(1+\alpha)}{2}-1\right]}{k^{2} \delta_{I P} \frac{\sigma_{\theta}^{2}}{\sigma^{2}}+1}, \text { when } \sigma_{I P}^{2} \rightarrow \infty
$$


substitute into (36), the expected project return is

$$
E\left[R^{I P} \mid \sigma_{I P}^{2} \rightarrow \infty\right]=\left[1-\frac{\beta^{2}\left[\frac{k(1+\alpha)}{2}-1\right]^{2}}{\left(k^{2} \delta_{I P} \frac{\sigma_{\theta}^{2}}{\sigma^{2}}+1\right)^{2}}\right] \cdot \theta_{0}
$$

Similarly, for a perfect input-based accounting, substituting $\sigma_{I P}^{2}=0$ into (19), and (20), we have $b_{z}^{I P}=1+\alpha$, and $b_{y}^{I P}=[2-(1+\alpha) k] \delta_{I P}^{-\frac{1}{2}}$. Then using $(22)$,

$$
\sqrt{\delta_{I P}}=1-\beta\left[\frac{k(1+\alpha)}{2}-1\right], \text { when } \sigma_{I P}^{2}=0
$$

substitute into (36), the expected project return is

$$
E\left[R^{I P} \mid \sigma_{I P}^{2}=0\right]=\left[1-\beta^{2}\left[\frac{k(1+\alpha)}{2}-1\right]^{2}\right] \cdot \theta_{0}
$$

Because, $\left(k^{2} \delta_{I P} \frac{\sigma_{\theta}^{2}}{\sigma^{2}}+1\right)^{2}>1$, so $E\left[R^{I P} \mid \sigma_{I P}^{2} \rightarrow \infty\right]>E\left[R^{I P} \mid \sigma_{I P}^{2}=0\right]$. Therefore, the expected project return in the basic setting is higher than the return with precise input-based reports. Because $\delta_{I P}$ is continuous in $\sigma_{I P}^{2}$, so that $E\left[R^{I P}\right]$ is continuous in $\sigma_{I P}^{2}$, there must exist a $\Sigma$ $\left(0<\Sigma<\sigma_{\theta}^{2}\right)$ such that for any $\sigma_{I P}^{2}>\Sigma\left(\sigma_{I P}^{2}<\Sigma\right)$, the input-based accounting is more (less) efficient than the basic cash flow reporting system.

To prove claim (iv), a similar argument shows that $E\left[R^{I P}\right]$ is single-peaked at $\sigma_{O P}^{2}=0$. Therefore, $E\left[R^{O P}\right]$ monotonically decreases in $\sigma_{O P}^{2} \in[0,+\infty)$ regardless of $k$. Given the following arguments:

- $E\left[R^{I P}\right]$ is strictly increasing in $\sigma_{I P}^{2} \in\left[0, \sigma_{\theta}^{2}\right]$ and reaches $E\left[R^{I P}\right]=1$ (the first-best) when $\sigma_{I P}^{2}=\sigma_{\theta}^{2}$;

- $E\left[R^{O P}\right]$ is strictly decreasing in $\sigma_{O P}^{2} \in\left[0, \sigma_{\theta}^{2}\right] \subset[0,+\infty)$ and reaches $E\left[R^{O P}\right]=1$ (the first-best) when $\sigma_{O P}^{2}=0$;

- Both $E\left[R^{I P}\right]$ and $E\left[R^{O P}\right]$ are continuous in $\sigma_{m}^{2}$ over $[0,+\infty)$;

there must exist a $\Sigma^{\prime}\left(\Sigma<\Sigma^{\prime}<\sigma_{\theta}^{2}\right)$, such that for any $\sigma_{I P}^{2} \in\left[\Sigma^{\prime}, \sigma_{\theta}^{2}\right]$ and $\sigma_{O P}^{2}>\Sigma^{\prime}, E\left[R^{I P}\right]>$ $E\left[R^{O P}\right]$, as claimed by (iv) of Theorem (4).

Proof of Theorem 5. After privately observing the unmanipulated $y^{m}$, and the realization of cash flow $\left(z_{1}\right)$ and cost parameter $\left(\xi^{m}\right)$, the manager/firm chooses the accounting report $w^{m}$ 
following the optimization program below, with an equilibrium conjecture of linear market pricing function $P\left(z_{1}, w^{m}\right)=a_{w}^{m}+b^{m} \times z_{1}+d^{m} \times w^{m}$, :

$$
\max _{w^{m}} \beta P\left(z_{1}, w^{m}\right)+(1-\beta) E_{z_{2}}\left[z_{1}+z_{2}\right]-c\left(w^{m}\right)
$$

Given the linear pricing and quadratic personal cost function, the solution to the optimization problem is well defined and yields a simple solution that in equilibrium, the following holds:

$$
w^{m}=y^{m}+\xi^{m}+\frac{\beta d^{m}}{c^{m}}
$$

Therefore the reported accounting number is simply a noised-up version of the underlying, unmanipulated number. Precisely, the reported accounting number has the following property:

$$
\begin{aligned}
E\left[w^{m}\right] & =E\left[y^{m}\right]+\frac{\beta d^{m}}{c^{m}} \\
\operatorname{Var}\left[w^{m}\right] & =\operatorname{Var}\left[y^{m}\right]+\operatorname{Var}\left[\xi^{m}\right]=\sigma_{m}^{2}+\eta_{m}^{2}
\end{aligned}
$$

The rest of the proof follows exactly as the proof of Theorem 3, substituting $v_{m}^{2}$ with $\operatorname{Var}\left[w^{m}\right]=$ $\sigma_{m}^{2}+\eta_{m}^{2}$. Note the adjustment to $E\left[y^{m}\right]$ will only affect the intercept part of the pricing function $\left(a_{w}^{m}\right)$, which has no incentive effects on the initial investment.

Proof of Corollary 2. Given Theorem 5, we know adding accounting manipulation option would increase the noise of the reported accounting number. Under the output-based accounting measures, given Corollary 1-(ii) and Theorem 4-(i), this leads to a loss of welfare on two fronts: (1) less efficient investment decisions and (2) non-zero (personal) cost of manipulation activities. so accounting manipulation always makes the firm worse off.

Under the input-based accounting measure, if $v_{I P}^{2}<\sigma_{\theta}^{2}$, adding noise to the accounting number improves investment efficiency, given Corollary 1-(ii) and Theorem 4 -(ii). If $c^{I P} \cdot \theta_{0}$ is high enough, the personal cost is small enough (because $c^{I P}$ is large) and the benefit of improved investment efficiency is large enough (because $\theta_{0}$ is large). So the investment benefit outweights the loss due to manipulation costs. Therefore, accounting manipulation is value-enhancing.

Proof of Theorem 6. Suppose the firm chooses an input-based accounting system, based on the linear conjecture:

$$
P\left(\lambda^{m}\right)=a_{\lambda}^{m}+b_{\lambda}^{m} \times \lambda^{m}
$$


the manager maximizes the expected payoff:

$$
\begin{aligned}
& \text { Choose } I_{1}(\theta) \text { to } \max \int_{\theta} V\left(\theta \mid I_{1}(\cdot)\right) G(\theta) d \theta \\
& =\int_{\theta} E\left[-I_{1}+\beta P+(1-\beta)\left(2 \sqrt{\theta I_{1}}+r \theta\right)\right] G(\theta) d \theta \\
& =-I_{1}+\beta\left(a_{\lambda}^{I P}+b_{\lambda}^{I P} I_{1}\right)+(1-\beta)\left(\int_{\theta}\left(2 \sqrt{\theta I_{1}}+r \theta\right) G(\theta) d \theta\right)
\end{aligned}
$$

The point-wise first-order condition wrt $I_{1}$ is for $\theta>0$, we have

$$
\begin{aligned}
0 & =-1+\frac{(1-\beta) \sqrt{\theta}}{\sqrt{I}}+\beta b_{\lambda}^{I P} \\
I_{1}^{I P} & =\left(\frac{1-\beta}{1-\beta b_{\lambda}^{I P}}\right)^{2} \theta
\end{aligned}
$$

and for $\theta<0, I_{1}^{I P}=0$. So it must be the case that

$$
\gamma_{I P}^{\prime}=\left(\frac{1-\beta}{1-\beta b_{\lambda}^{I P}}\right)^{2}
$$

Using (30), we have

$\left(\begin{array}{c}2 \sqrt{\gamma_{I P}^{\prime}} \theta+r \theta \\ \gamma_{I P}^{\prime} \theta+\varepsilon^{I P}\end{array}\right) \sim N\left(\left(\begin{array}{c}\left(2 \sqrt{\gamma_{I P}^{\prime}}+r\right) \theta_{0} \\ \gamma_{I P}^{\prime} \theta_{0}\end{array}\right),\left[\begin{array}{cc}\left(2 \sqrt{\gamma_{I P}^{\prime}}+r\right)^{2} \sigma_{\theta}^{2} & \left(2 \sqrt{\gamma_{I P}^{\prime}}+r\right) \gamma_{I P}^{\prime} \sigma_{\theta}^{2} \\ \left(2 \sqrt{\gamma_{I P}^{\prime}}+r\right) \gamma_{I P}^{\prime} \sigma_{\theta}^{2} & \left(\gamma_{I P}^{\prime}\right)^{2} \sigma_{\theta}^{2}+\sigma_{I P}^{2}\end{array}\right]\right)$

Using the approximation assumption, the pricing function becomes

$$
\begin{aligned}
P & =E\left[2 \sqrt{\theta I_{1}}+r \theta \mid \lambda^{I P}\right]=E\left[2 \sqrt{\theta I_{1}}+r \theta \mid I_{1}+\varepsilon^{I P}\right] \\
& \simeq E\left[\left(2 \sqrt{\gamma_{I P}^{\prime}}+r\right) \theta \mid \gamma_{I P}^{\prime} \theta+\varepsilon^{I P}\right] \\
& =\left(2 \sqrt{\gamma_{I P}^{\prime}}+r\right) \theta_{0}+\frac{\left(2 \sqrt{\gamma_{I P}^{\prime}}+r\right) \gamma_{I P}^{\prime} \sigma_{\theta}^{2}}{\left(\gamma_{I P}^{\prime}\right)^{2} \sigma_{\theta}^{2}+\sigma_{I P}^{2}}\left(\lambda^{I P}-\gamma_{I P}^{\prime} \theta_{0}\right)
\end{aligned}
$$

So it must be the case that

$$
\begin{aligned}
& b_{\lambda}^{I P}=\frac{\left(2 \sqrt{\gamma_{I P}^{\prime}}+r\right) \gamma_{I P}^{\prime} \sigma_{\theta}^{2}}{\left(\gamma_{I P}^{\prime}\right)^{2} \sigma_{\theta}^{2}+\sigma_{I P}^{2}} \\
& a_{\lambda}^{I P}=\left(2 \sqrt{\gamma_{I P}^{\prime}}+r-\gamma_{I P}^{\prime} b_{\lambda}^{I P}\right) \theta_{0}
\end{aligned}
$$

To show the existence of $\gamma_{I P}^{\prime}$ and $b_{\lambda}^{I P}$, substituting $b_{\lambda}^{I P}$ into $\gamma_{I P}^{\prime}$, and simplifying, we have

$$
\sqrt{\gamma_{I P}^{\prime}}-1=\beta \frac{\gamma_{I P}^{\prime}\left(2 \sqrt{\gamma_{I P}^{\prime}}+r-\gamma_{I P}^{\prime}\right)-\frac{\sigma_{I P}^{2}}{\sigma_{\theta}^{2}}}{\gamma_{I P}^{\prime}\left[\gamma_{I P}^{\prime}-\beta\left(2 \sqrt{\gamma_{I P}^{\prime}}+r\right)\right]+\frac{\sigma_{I P}^{2}}{\sigma_{\theta}^{2}}}
$$


As $\gamma_{I P}^{\prime} \in(0,+\infty)$, and the all functions are continuous, the left-hand-side is increasing in $\gamma_{I P}^{\prime}$ and covers the range from -1 to $+\infty$. The right-hand-side is $-\beta$ as $\gamma_{I P}^{\prime}$ is zero, and also converges to $-\beta$ as $\gamma_{I P}^{\prime}$ goes to infinity. Since we assume that $\beta$ is from -1 to 1 . So the left-hand-side curve and the right- hand-side curve must intersect at least once in $\gamma_{I P}^{\prime} \in(0,+\infty)$. Therefore, there is at least one positive root of $\gamma_{I P}^{\prime}$.

Using the similar method, we obtain the linear equilibrium under output-based accounting system.

Proof of Corollary 3. Suppose the manager chooses the first-best investment level $I_{1}=\theta$ under input-based accounting. Correspondingly, using (29), the market pricing function would be

$$
\begin{aligned}
P\left(\lambda^{I P}\right) & =a_{\lambda}^{I P}+b_{\lambda, \gamma_{I P}^{\prime}=1}^{I P} \times \lambda^{I P}, \text { where } \\
b_{\lambda, \gamma_{I P}^{\prime}=1}^{I P} & =\frac{(2+r) \sigma_{\theta}^{2}}{\sigma_{\theta}^{2}+\sigma_{I P}^{2}}
\end{aligned}
$$

To sustain the equilibrium, the manager's reaction to $b_{\lambda, \gamma_{I P}^{\prime}=1}^{I P}$ must, indeed, be to set the investment at the first-best level. That is, it must be the case that

$$
\left.\gamma_{I P}^{\prime}\right|_{b_{\lambda, \gamma_{I P}^{I P}=1}^{I P}}=1
$$

By assuming $\sigma_{I P}^{2}=(r+1) \sigma_{\theta}^{2}$, we have

$$
b_{\lambda, \gamma_{I P}^{\prime}=1}^{I P}=\frac{(2+r) \sigma_{\theta}^{2}}{\sigma_{\theta}^{2}+\sigma_{I P}^{2}}=1
$$

Using (30), we have

$$
\gamma_{I P}^{\prime}=\left(\frac{1-\beta}{1-\beta b_{\lambda, \gamma_{I P}^{\prime}=1}^{I P}}\right)^{2}=1
$$

Using the similar method, we get the firm is motivated to choose $\gamma_{O P}^{\prime}=1$ if $\sigma_{O P}^{2}=2 r \sigma_{\theta}^{2}$ under output-based accounting. As the investment $\gamma_{m}^{\prime}$ is strictly decreasing in the measurement noise $\sigma_{m}^{2}$, under both accounting measurements, the efficiency of investment is strictly decreasing from the first-best to either direction. As $\gamma_{m}^{\prime}$ is continuous in $\sigma_{m}^{2}$, if $r>1(r<1)$, there must exist a $\Sigma^{*}$ which lies in the interval of $(r+1) \sigma_{\theta}^{2}$ and $2 r \sigma_{\theta}^{2}$, such that input-based accounting is more efficient than output-based accounting when $\sigma_{I P}^{2}=\sigma_{O P}^{2} \in\left[(r+1) \sigma_{\theta}^{2}, \Sigma^{*}\right]\left(\sigma_{I P}^{2}=\sigma_{O P}^{2} \in\left[\Sigma^{*},(r+1) \sigma_{\theta}^{2}\right]\right)$. 


\section{References}

[1] Christensen, J., and J. S. Demski, 2002. Accounting Theory: an information content perspective. McGraw-Hill.

[2] Demski, Joel, Haijin Lin, and David E. M. Sappington, 2005 Asset Impairment Regulations. University of Florida Working paper.

[3] Dye, R. A., and S. S. Sridhar. 2004a. Reliability-Relevance Trade-offs and the Efficiency of Aggregation. Journal of Accounting Research 42: 51-87.

[4] Dye, R. A. 2002. Classifications Manipulation and Nash Accounting Standards. Journal of Accounting Research 40:1125-1162.

[5] Dye, R. A., and S. S. Sridhar. 2004b. A Simple Model of The Costs and Benefits of Accounting Standards, working paper.

[6] Edwards, E. O., and P. W. Bell. 1961. The Theory of Measurement of Business Income. Berkeley, CA: University of California Press.

[7] Financial Accounting Standard Board. 2004. Fair Value Measurements (Proposed Statement of Financial Accounting Standards). Norwalk, CT: Financial Accounting Standards Board.

[8] Holmstrm, B. 1982. Managerial Incentives Problems - A Dynamic Perspective. Originally written for an unpublished volume Essays in Economics and Management in Honour of Professor Lars Wahlback. Helsinki: Swedish School of Economics and Business Administration. Published in 1999 in Review of Economic Studies 66 (1):169-182.

[9] Ijiri, Yuji. 1975. Theory of Accounting Measurement, Studies in Accounting Research \#10. Sarasota, FL: American Accounting Association.

[10] Kanodia, C., R. Singh, and A. Spero. 2005. Imprecision in Accounting Measurement: Can it be value enhancing? Journal of Accounting Research 43:487-519.

[11] Myers, S.. 1977. Determinants of Corporate Borrowing. Journal of Financial Economics vol. 5, p. $147-175$.

[12] William Paton and A. C. Littleton. 1940. An Introduction to Corporate Accounting Standards. AAA monograph

[13] Prendergast, C. 2002. The Tenuous Trade-off between Risk and Incentives. Journal of Political Economy 110: 1071-1102.

[14] Stein, J. 1989. Efficient capital markets, inefficient firms: A model of myopic corporate behavior. The Quarterly Journal of Economics 104: 655-670. 
[15] Sterling, R. R. 1970. Theory of the Measurement of Enterprise Income. Lawrence, KS: The University of Kansas Press.

[16] Stocken, P. C., and R. E. Verrecchia. 2004. Financial Reporting System Choice and Disclosure Management. The Accounting Review 79:1181-1203. 
Figure 2: The effect of accounting quality on the net project return

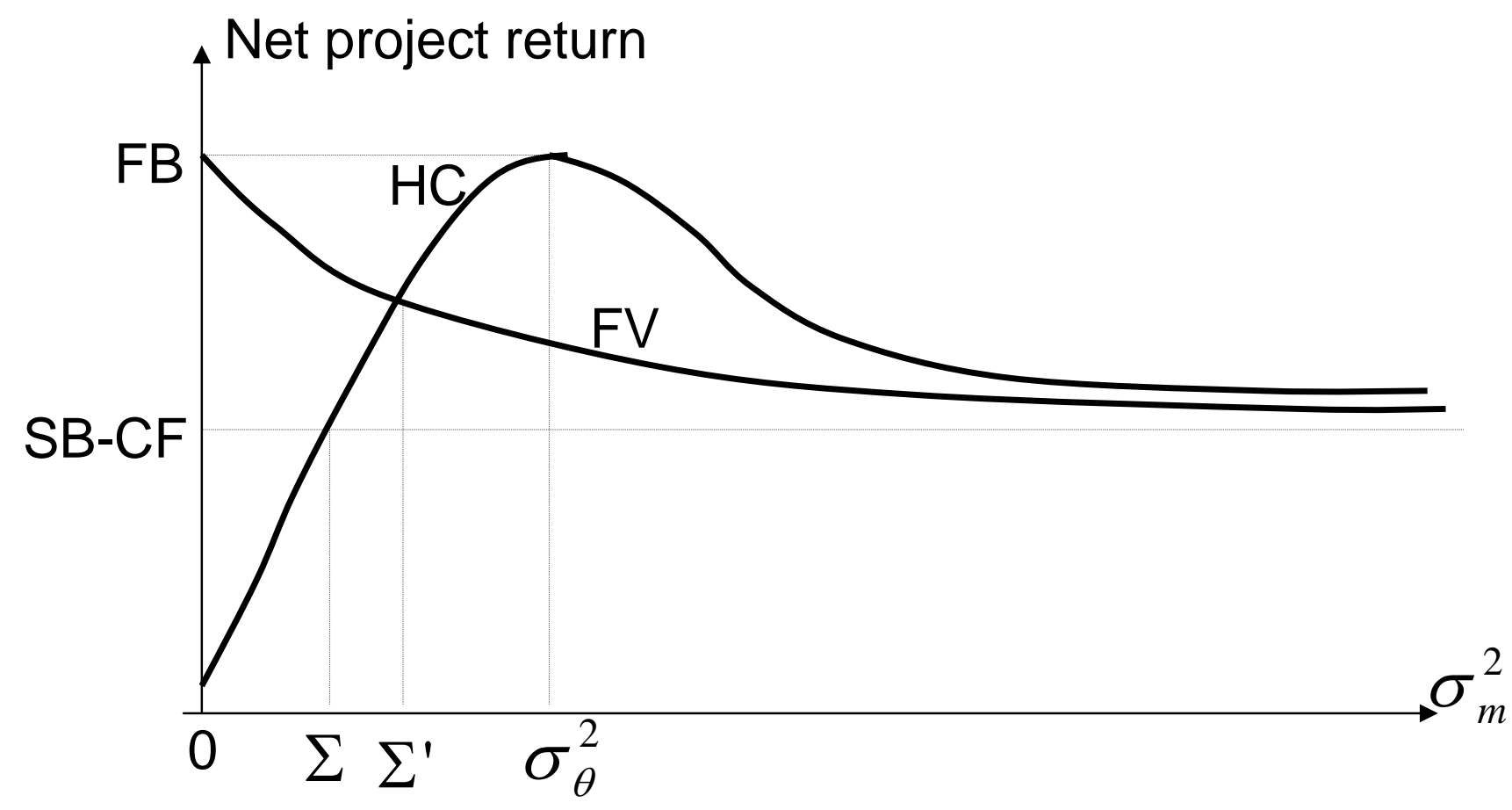


Figure 3: Investment Efficiency in the Modified Model $(r=2)$

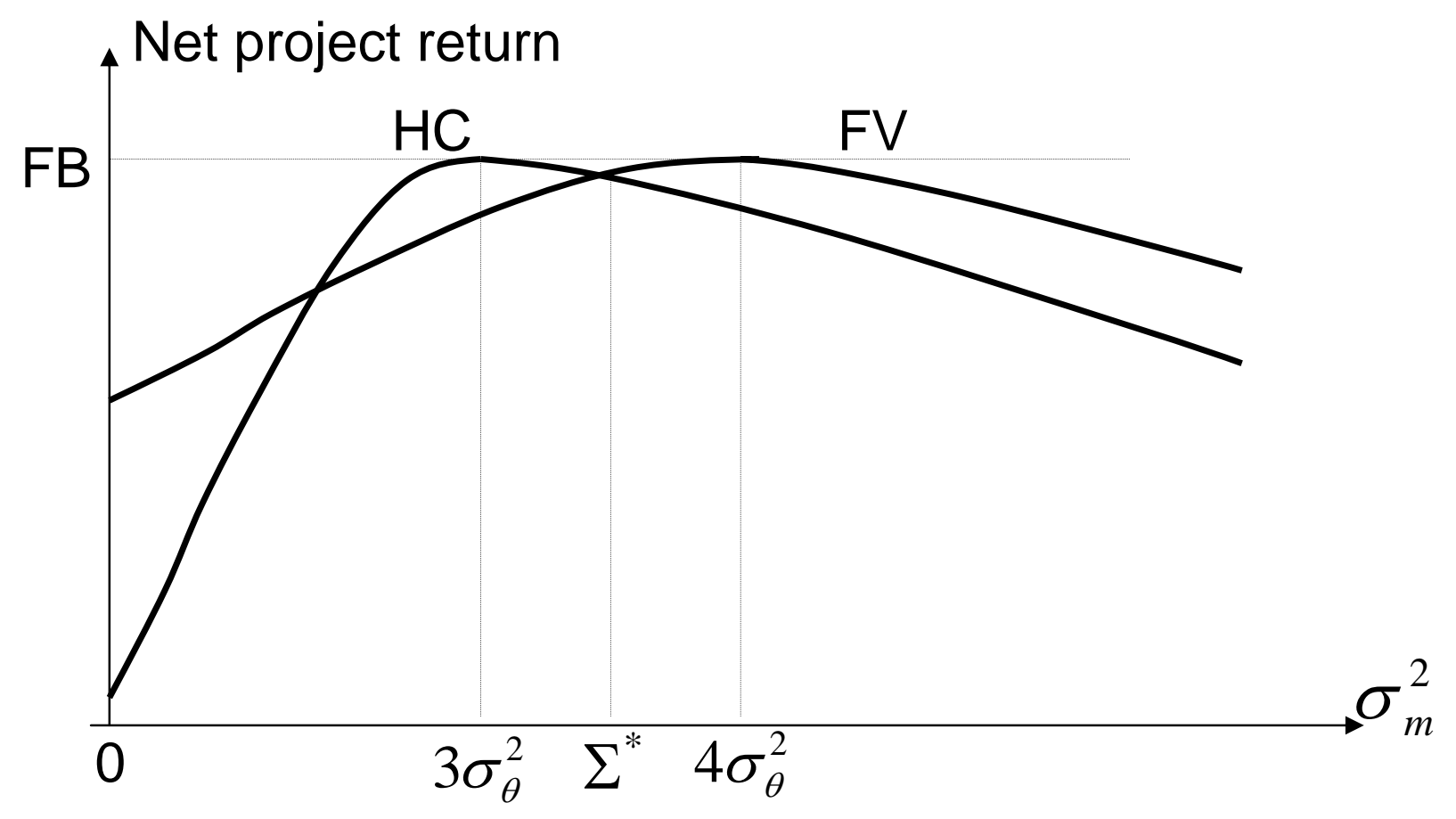

